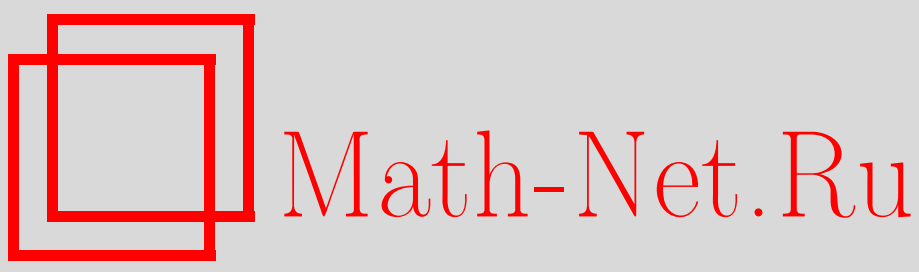

А. А. Милютин, Об усилении условий Кларка и Смирнова для выпуклозначных дифференциальных включений, Матем. сб., 2003, том 194, номер 2, 87-116

DOI: https://doi.org/10.4213/sm715

Использование Общероссийского математического портала Math-Net.Ru подразумевает, что вы прочитали и согласны с пользовательским соглашением http://www . mathnet.ru/rus/agreement

Параметры загрузки:

IP: 18.207 .199 .55

26 апреля 2023 г., 13:20:00 
А.А. Милютин

\title{
Об усилении условий Кларка и Смирнова для выпуклозначных дифференциальных включений
}

\begin{abstract}
На фиксированном отрезке времени задано липшицево дифференциальное включение с выпуклыми значениями и локально компактньм графикиом. Для траекторий этого включения рассматривается задача на минимум гладкой концевой функции при гладких концевых ограничениях равенства и неравенства. Эта задача приближается последовательностью гладких задач оптимального управления с регулярньми смешанными ограничениями, к которым применяется принцип максимума, полученный ранее автором совместно с А. Я. Дубовицким. $\mathrm{C}$ помощью предельного перехода в условиях принципа максимума получены необходимые условия сильного минимума в исходной задаче, усиливающие известные условия $\Phi$. Кларка и Г. Смирнова.

Библиографоя: 3 названия.
\end{abstract}

\section{Вводная часть}

\section{Введение}

Мы рассматриваем следуюшую задачу.

ЗАДАЧА $Z$.

$$
\begin{gathered}
J(p) \rightarrow \min , \quad h(p) \leqslant 0, \quad g(p)=0, \\
\dot{x} \in F(x, t), \quad t \in\left[t_{0}, t_{1}\right] .
\end{gathered}
$$

Здесь $p=\left(x_{0}, x_{1}\right), x_{0}=x\left(t_{0}\right), x_{1}=x\left(t_{1}\right), x \in \mathbb{R}^{d(x)}, h \in \mathbb{R}^{d(h)}$, через $d(h)$ обозначается размерность вектора $h$. Функции $J, h, g$ непрерывно дифференцируемы во всем пространстве $\mathbb{R}^{d(p)}$, многозначное отображение $F$ выпуклозначно, обладает локально компактньм графиком, ограничено во всем пространстве $\mathbb{R}^{d(x)+1}$ и удовлетворяет условию Липшица по $x, t$ относительно расстояния по Хаусдорфу.

Мы получим новые, до сих пор неизвестные необходимые условия сильного минимума в задаче $Z$. Мы будем опираться при этом не на негладкий анализ, а на принцип максимума (далее ПМ) для задач с регулярньми смешанными ограничениями, найденньй Дубовицким и Милютиньм [1].

В отличие от Кларка [2], мы нигде не использовали принцип Экланда, а заменили его обычньм штрафом.

Работа вьполнена при поддержке Российского фонда фундаментальных исследований (грант № 00-15-96109).

(C) А.А. Милютин 2003 
Насколько нам известно, ПМ для задач со смешанными ограничениями ранее никем не использовался при исследовании задач оптимизации, поставленных для дифференциальных включений. Мы не знаем, насколько успешно можно применять негладкий анализ для получения наших необходимых условий.

В работе приняты следуюшие обозначения:

$x y$ - скалярное произведение векторов $x, y \in \mathbb{R}^{n}$;

$|x|$ - евклидова норма вектора $x \in \mathbb{R}^{n}$;

$п_{b} a=a b /|b|-$ проекция вектора $a$ на направление вектора $b$;

$\left(\mathbb{R}^{n}\right)^{*}$ - пространство вектор-строк размерности $n$;

$\overline{\mathrm{lt}}_{n} A_{n}$ - верхний топологический предел последовательности множеств $A_{n} \subset \mathbb{R}^{n}$; $\operatorname{cl} Q$ - замыкание множества $Q \subset \mathbb{R}^{n}$;

conv $Q$ - вьпуклая оболочка множества $Q$;

$\operatorname{dist}(A, B)$ - расстояние по Хаусдорфу между множествами $A$ и $B$ в $\mathbb{R}^{n}$.

Для сублинейного (т.е. выпуклого и положительно однородного) функционала $\varphi(x)$ через $\partial \varphi$ обозначается его субдифференциал (или множество опорных) в нуле, состоящий из всех линейных функционалов $l$ таких, что $\langle l, x\rangle \leqslant \varphi(x) \forall x$.

Если функция $\Phi(x, y)$ является сублинейной по переменной $x$, то $\partial_{x} \Phi(0, y)$ есть субдифференциал по этой переменной в нуле при фиксированном $y$.

Для функции $f: \mathbb{R}^{n} \rightarrow \mathbb{R}$ через $\stackrel{\circ}{f}(x, \bar{x})$, а также через $f_{x}(x, \bar{x})$ обозначается ее производная в точке $x$ по направлению $\bar{x}$, а через $\stackrel{\circ}{f}_{\text {вер }}(x, \bar{x})$ - верхняя производная этой функции в точке $x$ по направлению $\bar{x}$, определяемая равенством

$$
\stackrel{\circ}{f \text { вер }}(x, \bar{x})=\varlimsup_{\varepsilon \rightarrow+0} \frac{f(x+\varepsilon \bar{x})-f(x)}{\varepsilon} .
$$

Наконец, $\stackrel{\circ}{\mathrm{Cl}}(x, \bar{x})$ есть производная Кларка в точке $x$ по направлению $\bar{x}$, определяемая равенством

$$
\stackrel{\circ}{f}_{\mathrm{Cl}}(x, \bar{x})=\varlimsup_{\substack{x^{\prime} \rightarrow x \\ \varepsilon \rightarrow+0}} \frac{f\left(x^{\prime}+\varepsilon \bar{x}\right)-f\left(x^{\prime}\right)}{\varepsilon} .
$$

Через $f^{\gamma}(x)$ обозначается осреднение функции $f(x)$ с ядром осреднения $\gamma=\gamma(x)$; через $\eta(\gamma)$ обозначается радиус носителя функции $\gamma$.

Запись вида $\mathscr{A} \mid \mathscr{B}$ означает, что условие $\mathscr{A}$ выполняется при выполнении условия $\mathscr{B}$.

\section{§1. Некоторые предварительные сведения}

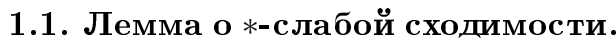

ЛЕмма 1.1. Пусть последовательность $\xi^{n}(t)$ вектор-функций из $L_{\infty}\left[t_{0}, t_{1}\right]$ *-слабо сходится $\kappa \xi^{0}(t)$. Тогда существует множсество Е полной меры на $\left[t_{0}, t_{1}\right]$, в кажсдой точке которого для любого $\bar{\xi} \in \mathbb{R}^{d(\xi)}$ выполнено неравенствo

$$
\xi^{0}(t) \bar{\xi} \leqslant \varlimsup_{n \rightarrow \infty} \xi^{n}(t) \bar{\xi}
$$


ДокАЗАТЕЛЬСТво. По условию для любой вектор-функции $\bar{\xi}(t)$ из $L_{1}\left[t_{0}, t_{1}\right]$

$$
\int_{t_{0}}^{t_{1}} \xi^{0}(t) \bar{\xi}(t) d t=\lim _{n \rightarrow \infty} \int_{t_{0}}^{t_{1}} \xi^{n}(t) \bar{\xi}(t) d t
$$

следовательно,

$$
\int_{t_{0}}^{t_{1}} \xi^{0}(t) \bar{\xi}(t) d t \leqslant \int_{t_{0}}^{t_{1}} \varlimsup_{n \rightarrow \infty} \xi^{n}(t) \bar{\xi}(t) d t
$$

Отсюда для любых $t^{\prime}<t^{\prime \prime}$ из $\left[t_{0}, t_{1}\right]$ и для любого вектора $\bar{\xi} \in \mathbb{R}^{d(\xi)}$

$$
\int_{t^{\prime}}^{t^{\prime \prime}} \xi^{0}(t) \bar{\xi}(t) d t \leqslant \int_{t^{\prime}}^{t^{\prime \prime}} \varlimsup_{n \rightarrow \infty} \xi^{n}(t) \bar{\xi}(t) d t
$$

Тогда для любого $\bar{\xi}$ сушествует множество полной меры, в каждой точке которого выполнено (1.1). Пересекая эти множества, соответствующие счетному всюду плотному множеству векторов $\bar{\xi}$, получаем требуемое множество $\mathscr{E}$.

\section{2. Дифференциальное включение.}

ПРЕДЛОЖЕНИЕ 1.1. Пусть имеется равномерно ограниченная последовательность выпуклозначных отображсений $F^{n}(x, t), n=1,2, \ldots$, такая, что

$$
\overline{\mathrm{lt}}_{n} F^{n}(x, t) \subset F(x, t) \quad \forall(x, t) .
$$

Пусть для любого $n$ липшицева функиия $x^{n}(t)$ удовлетворяет п.в. на $\left[t_{0}, t_{1}\right]$ дифференциальному включению $\dot{x} \in F^{n}(x, t)$, и пусть $x^{0}(t)$ - предельная точка последовательности $x^{n}(t)$ (относительно равномерной или, что в данном случае әквивалентно, относительно поточечной сходимости). Тогда $x^{0}(t)$ удовлетворяет п.в. на $\left[t_{0}, t_{1}\right]$ дифференииальному включению $\dot{x} \in F(x, t)$.

ДоказАтЕльство. Так как равномерная сходимость $x^{n}(\cdot) \rightarrow x(\cdot)$ влечет сходимость $\dot{x}^{n}(\cdot) \stackrel{* \text {-сл }}{\longrightarrow} \dot{x}^{0}(\cdot)$, то по лемме 1.1 на множестве полной меры $\mathscr{E}$ выполнено неравенство

$$
\bar{x} \dot{x}^{0}(t) \leqslant \varlimsup_{n \rightarrow \infty} \bar{x} \dot{x}^{n}(t) \quad \forall \bar{x}
$$

Отсюда

$$
\dot{x}^{0}(t) \in \operatorname{conv} \underset{n}{\overline{\operatorname{lt}}} F^{n}\left(x^{n}(t), t\right) \text { на } \mathscr{E} .
$$

Следовательно,

$$
\dot{x}^{0}(t) \in F\left(x^{0}(t), t\right) \text { на } \mathscr{E} .
$$

Предложение доказано.

Мы будем говорить, что последовательность вьпуклозначных отображений $F^{n}(x, t)$ приближается $\kappa F(x, t)$, если она равномерно ограничена и выполнено условие (1.2). 


\section{§2. Теоремы приближаемости}

Пусть $F^{n}(x, t)$ приближается к $F(x, t)$, причем $F^{n}(x, t) \supset F(x, t) \forall n, x, t$. Будем называть это приближением сверху.

Пусть $x^{0}(t)$ есть точка сильного минимума задачи $Z$.

2.1. Первая теорема приближаемости. Рассмотрим задачу $Z^{n}$ :

$$
\begin{gathered}
J(p)+\int_{t_{0}}^{t_{1}}\left(x(t)-x^{0}(t)\right)^{2} d t \rightarrow \min , \\
h(p) \leqslant 0, \quad g(p)=0 \\
\dot{x} \in F^{n}(x), \quad t \in\left[t_{0}, t_{1}\right] .
\end{gathered}
$$

Tеорема 2.1. Существует последовательность $x^{n}(t)$ траекторий задачи $Z^{n}$, определенная для всех достаточно больших $n$, такая, что $x^{n}(t)$ есть сильный минимум задачи $Z^{n}$ и $x^{n}(t) \rightarrow x^{0}(t)$ равномерно на $\left[t_{0}, t_{1}\right]$.

ДоказАТЕЛЬство. Так как $x^{0}(t)$ есть сильный минимум в задаче $Z$, сушествует ограниченное открытое множество $Q \in \mathbb{R}^{d(x)+1}$ такое, что $\left(x^{0}(t), t\right) \in Q$ $\forall t \in\left[t_{0}, t_{1}\right]$ и $x^{0}(t)$ есть абсолютный минимум в задаче $Z_{Q}$, которая получена из задачи $Z$ добавлением ограничения $(x, t) \in \mathrm{cl} Q$. Соответственно, рассмотрим последовательность задач $Z_{Q}^{n}, n=1,2, \ldots$. Для любого $n$ задача $Z_{Q}^{n}$ имеет решение. Обозначим его через $x^{n}(t)$. Очевидно, последовательность $x^{n}(t)$ предкомпактна в норме $C\left[t_{0}, t_{1}\right]$. Пусть $\widehat{x}(t)$ есть ее предельная точка. Согласно предложению 1.1 она удовлетворяет дифференциальному включению

$$
\frac{d}{d t} \widehat{x} \in F(\widehat{x}(t), t) .
$$

Но значение задачи $Z_{Q}^{n}$ не превосходит $J\left(p^{0}\right)$, следовательно,

$$
J(\widehat{p})+\int_{t_{0}}^{t_{1}}\left(\widehat{x}(t)-x^{0}(t)\right)^{2} d t \leqslant J\left(p^{0}\right) .
$$

$\mathrm{C}$ другой стороны, $\widehat{x}(t)$ есть допустимая траектория задачи $Z_{Q}$, поэтому $J(\widehat{p}) \geqslant$ $J\left(p^{0}\right)$. Отсюда $\widehat{x}(t)=x^{0}(t)$. Теорема доказана.

2.2. Вторая теорема приближаемости. Для любого $n$ определим следующую задачу $\widetilde{Z}^{n}$ :

$$
\begin{gathered}
J(p)+\left(p-p^{0}\right)^{2}+\int_{t_{0}}^{t_{1}}\left(\dot{x}(t)-\dot{x}^{0}(t)\right)^{2} d t \rightarrow \min , \\
h(p) \leqslant 0, \quad g(p)=0 \\
\dot{x} \in F^{n}(x), \quad t \in\left[t_{0}, t_{1}\right] .
\end{gathered}
$$

ТЕОРема 2.2. Начиная с некоторого номера $n$ существует траектория $\widetilde{x}^{n}(t)$ - сильныц минимум задачи $\widetilde{Z}^{n}$, такая, что $\widetilde{x}^{n}(t) \rightarrow x^{0}(t)$ равномерно на $\left[t_{0}, t_{1}\right] u$

$$
\int_{t_{0}}^{t_{1}}\left(\dot{\widetilde{x}}^{n}(t)-\dot{x}^{0}(t)\right)^{2} d t \rightarrow 0, \quad n \rightarrow \infty .
$$

Доказательство этой теоремы практически не отличается от доказательства предыдущей. 


\section{§3. Два типа аппроксимирующих последовательностей}

Следуя Кларку, введем функцию

$$
\mathscr{H}(\psi, x, t)=\max \{\psi u \mid u \in F(x, t)\}
$$

- гамильтониан отображения $F(x, t)$. Имеется очевидная оценка:

$$
\left|\mathscr{H}\left(\psi, x_{1}, t_{1}\right)-\mathscr{H}\left(\psi, x_{2}, t_{2}\right)\right| \leqslant c_{0}|\psi|\left|\left(x_{1}, t_{1}\right)-\left(x_{2}, t_{2}\right)\right|,
$$

где $c_{0}$ - постоянная Липшица отображения $F$. Верно и обратное утверждение: если гамильтониан удовлетворяет (3.1), то $F(x, t)$ удовлетворяет условию Липшица с постоянной $c_{0}$.

Гамильтониан всегда сублинеен по $\psi$, и для всех $(x, t)$

$$
F(x, t)=\partial_{\psi} \mathscr{H}(0, x, t)
$$

есть его субдифференциал по $\psi$.

Рассмотрим также $\rho(x, t, u)=\operatorname{dist}(u, F(x, t))$ - функцию расстояния отображения $F(x, t)$. Так как множество $F(x, t)$ выпукло для всех $(x, t)$, то функция расстояния выпукла по $u$, причем расстояние от $u$ до $F(x, t)$ всегда реализуется в одной точке. Отсюда следует, что функция $\rho(x, t, u)$ имеет непрерывную производную по $u$ в каждой точке, где $\rho(x, t, u)>0$, причем в такой точке $\left|\rho_{u}(x, t, u)\right|=1$.

Между гамильтонианом и функцией расстояния имеется связь (формула двойственности):

$$
\rho(x, t, u)=\max \{\psi u-\mathscr{H}(\psi, x, t)|| \psi \mid \leqslant 1\} .
$$

Если $\rho(x, t, u)>0$, то максимум здесь достигается в единственной точке $\psi=$ $\rho_{u}(x, t, u)$ и поэтому

$$
\rho(x, t, u)=\rho_{u}(x, t, u) u-\mathscr{H}\left(\rho_{u}(x, t, u), x, t\right) .
$$

Отсюда следует, что если гамильтониан имеет непрерьвную производную по $x$, то функция расстояния также имеет непрерьвную производную по $x$ в каждой точке, в которой $\rho(x, t, u)>0$, причем

$$
\rho_{x}(x, t, u)=-\mathscr{H}_{x}\left(\rho_{u}(x, t, u), x, t\right) .
$$

Аналогично, если гамильтониан имеет непрерьвную производную по $t$, то функция расстояния также имеет непрерьвную производную по $t$ в каждой точке, в которой $\rho(x, t, u)>0$, причем

$$
\rho_{t}(x, t, u)=-\mathscr{H}_{t}\left(\rho_{u}(x, t, u), x, t\right) .
$$

3.1. Осреднение гамильтониана. Пусть $\gamma(x, t)$ есть ядро осреднения по $x, t$, т.е. финитная гладкая неотрицательная функция такая, что $\int \gamma(x, t) d x d t=1$, где интеграл берется по всему пространству $\mathbb{R}^{d(x)+1}$. Пусть

$$
\eta(\gamma)=\min \{\eta \mid \gamma(x, t)=0 \text { при }|(x, t)| \geqslant \eta\}
$$


- радиус носителя функции $\gamma$. Рассмотрим функцию

$$
\mathscr{H}^{\gamma}(\psi, x, t)=\int d x^{\prime} d t^{\prime}\left\{\mathscr{H}\left(\psi, x^{\prime}, t^{\prime}\right) \gamma\left(x^{\prime}-x, t^{\prime}-t\right)\right\}
$$

- осреднение функиии $\mathscr{H}$ с помощъю ядра $\gamma$. Отметим, что $\mathscr{H}^{\gamma}$ по-прежнему сублинейна по $\psi$. Из (3.1) следует оценка:

$$
\left|\mathscr{H}^{\gamma}\left(\psi, x_{1}, t_{1}\right)-\mathscr{H}^{\gamma}\left(\psi, x_{2}, t_{2}\right)\right| \leqslant|\psi| c_{0}\left|\left(x_{1}, t_{1}\right)-\left(x_{2}, t_{2}\right)\right|
$$

Имеем также

$$
\left|\mathscr{H}^{\gamma}(\psi, x, t)-\mathscr{H}(\psi, x, t)\right| \leqslant c_{0} \eta(\gamma)|\psi|
$$

Определим многозначное отображение $\bar{F}^{\gamma}(x, t)=\partial_{\psi} \mathscr{H}^{\gamma}(0, x, t)$ и соответствующую ему функцию $\bar{\rho}^{\gamma}(x, t, u)=\operatorname{dist}\left(u, \bar{F}^{\gamma}(x, t)\right)$.

Из (3.7) следует, что расстояние по Хаусдорфу

$$
\operatorname{dist}\left(\bar{F}^{\gamma}(x, t), F(x, t)\right) \leqslant c_{0} \eta(\gamma)
$$

Согласно (3.3)-(3.5) функция $\bar{\rho}^{\gamma}$ обладает непрерывным полньм дифференциалом по $x, t, u$ в каждой точке, где $\bar{\rho}^{\gamma}>0$.

Определим множество

$$
\bar{U}^{\gamma}(x, t)=\left\{u \mid \bar{\rho}^{\gamma}(x, t, u) \leqslant c_{0} \eta(\gamma)\right\}
$$

Из (3.8) следует, что для всех $(x, t)$ справедливо включение $\bar{U}^{\gamma}(x, t) \supset F(x, t)$ и

$$
\overline{\mathrm{lt}}\left\{\bar{U}^{\gamma}(x, t) \mid \eta(\gamma) \rightarrow 0\right\}=F(x, t)
$$

3.2. Осреднение функции расстояния. Для упрощения записи введем обозначение $y=(x, t)$.

ПРЕДЛОЖЕНИЕ 3.1. Пусть $y_{1}, y_{2} u \delta>0$ maковь, что $\left|y_{1}-y_{2}\right| \leqslant \delta, u$ $0<\varepsilon<1$. Пусть и удовлетворяет условию

$$
c_{0} \delta \leqslant \varepsilon \cdot \max \left\{\rho\left(y_{1}, u\right), \rho\left(y_{2}, u\right)\right\}
$$

әде с $c_{0}$ - константа Липиица отображсения $F$ ( а следовательно, и функиии $\rho$ ) по у. Тогда имеет место неравенство

$$
\left|\rho_{u}\left(y_{1}, u\right)-\rho_{u}\left(y_{2}, u\right)\right| \leqslant \sqrt{2 \varepsilon}
$$


ДоКАЗАТЕЛЬСтво. Пусть для определенности $\rho\left(y_{1}, u\right) \geqslant \rho\left(y_{2}, u\right)$. Тогда из (3.10) следует, что $\rho\left(y_{2}, u\right) \geqslant(1-\varepsilon) c_{0} \delta / \varepsilon$.

Пусть $u_{1}$ и $u_{2}$ удовлетворяют условиям:

$$
\begin{array}{ll}
u_{1} \in F\left(y_{1}\right), & \left|u_{1}-u\right|=\rho\left(y_{1}, u\right), \\
u_{2} \in F\left(y_{2}\right), & \left|u_{2}-u\right|=\rho\left(y_{2}, u\right) .
\end{array}
$$

Так как $\operatorname{dist}\left(F\left(y_{2}\right), F\left(y_{1}\right)\right) \leqslant c_{0} \delta$, то в силу (3.10) получаем

$$
\left|u_{1}-u\right|-\operatorname{mp}_{\left(u_{1}-u\right)}\left(u_{2}-u\right) \leqslant \varepsilon\left|u_{1}-u\right| .
$$

Умножая на $\left|u_{1}-u\right|$, получаем отсюда

$$
\left|u_{1}-u\right|^{2}-\left(u_{1}-u\right)\left(u_{2}-u\right) \leqslant \varepsilon\left|u_{1}-u\right|^{2} .
$$

Положим $\Delta \rho_{u}=\rho_{u}\left(y_{1}, u\right)-\rho_{u}\left(y_{2}, u\right)$. Имеем

$$
\rho_{u}\left(y_{1}, u\right)=-\frac{u_{1}-u}{\left|u_{1}-u\right|}, \quad \rho_{u}\left(y_{2}, u\right)=-\frac{u_{2}-u}{\left|u_{2}-u\right|} .
$$

Согласно (3.12)

$$
\left(u_{1}-u\right)\left(u_{2}-u\right) \geqslant(1-\varepsilon)\left|u_{1}-u\right|^{2}
$$

откуда

$$
\frac{\left(u_{1}-u\right)\left(u_{2}-u\right)}{\left|u_{1}-u\right|^{2}} \geqslant 1-\varepsilon .
$$

Но $\left|u_{1}-u\right|=\rho\left(y_{1}, u\right) \geqslant \rho\left(y_{2}, u\right)=\left|u_{2}-u\right|$. Следовательно,

$$
\frac{\left(u_{1}-u\right)\left(u_{2}-u\right)}{\left|u_{1}-u\right| \cdot\left|u_{2}-u\right|} \geqslant 1-\varepsilon .
$$

Отсюда и из (3.13) следует $\left|\Delta \rho_{u}\right|^{2} \leqslant 2 \varepsilon$. Предложение доказано.

Далее, рассмотрим функцию

$$
\rho^{\gamma}(y, u)=\int d y^{\prime}\left\{\rho\left(y^{\prime}, u\right) \gamma\left(y^{\prime}-y\right)\right\}
$$

Очевидно,

$$
\begin{gathered}
\left|\rho^{\gamma}(y, u)-\rho(y, u)\right| \leqslant c_{0} \eta(\gamma) \\
\left|\rho^{\gamma}\left(y_{1}, u\right)-\rho^{\gamma}\left(y_{2}, u\right)\right| \leqslant c_{0}\left|y_{1}-y_{2}\right| \forall y_{1}, y_{2}, u
\end{gathered}
$$

ПРЕДЛОЖЕНИЕ 3.2. Пусть $0<\varepsilon<1 / 2$, число $\eta$ таково, что

$$
c_{0} \eta(\gamma) \frac{1+\varepsilon}{\varepsilon} \leqslant \eta
$$

и точки у, и таковы, что

$$
\rho^{\gamma}(y, u)=\eta
$$

Тогда

$$
\left|\rho_{u}^{\gamma}(y, u)-\rho_{u}(y, u)\right| \leqslant \sqrt{2 \varepsilon}
$$


ДокаЗАТЕЛЬСтво. Из (3.14a) и (3.15b) имеем $\rho(y, u) \geqslant \eta-c_{0} \eta(\gamma)$. Но согласно $(3.15 \mathrm{a}) \eta-c_{0} \eta(\gamma) \geqslant c_{0} \eta(\gamma) / \varepsilon$. Следовательно, $c_{0} \eta(\gamma) \leqslant \varepsilon \rho(y, u)$. Из предложения 3.1 при $\delta=c_{0} \eta(\gamma)$ следует

$$
\left|\rho_{u}\left(y^{\prime}, u\right)-\rho_{u}(y, u)\right| \leqslant \sqrt{2 \varepsilon} \forall y^{\prime}:\left|y^{\prime}-y\right| \leqslant c_{0} \eta(\gamma)
$$

Отсюда легко вытекает требуемое неравенство.

Поскольку $\left|\rho_{u}(y, u)\right|=1$ при $\rho(y, u)>0$, то имеет место

СлЕДСТВИЕ. В условиях предложения 3.2 выполнено неравенство

$$
\left|\rho_{u}^{\gamma}(y, u)\right| \geqslant 1-\sqrt{2 \varepsilon}
$$

ПРЕДЛОЖЕНИЕ 3.3. Пусть последовательности $\gamma^{n}(y), \eta^{n}$ таковы, что $\eta^{n} \rightarrow 0$ и $\eta\left(\gamma^{n}\right)=o\left(\eta^{n}\right)$. Определим множество

$$
U^{n}(y)=\left\{u \mid \rho^{\gamma^{n}}(y, u) \leqslant \eta^{n}\right\}
$$

Тогда начиная с некоторого $n \quad U^{n}(y) \supset F(y) u$

$$
\bar{n} U^{n}(y)=F(y) \text {. }
$$

Доказательство элементарно следует из (3.14).

\section{Часть І. Усиление условий Кларка}

\section{§4. Аппроксимации и принцип максимума}

4.1. Аппроксимируюшая последовательность. Пусть заданы последовательности $\nu^{m}(t), \mu^{n}(x)$ ядер осреднения по $t$ и $x$ соответственно, причем $\eta\left(\nu^{m}\right) \rightarrow 0, \eta\left(\mu^{n}\right) \rightarrow 0$. Положим $\gamma^{n, m}(x, t)=\mu^{n}(x) \nu^{m}(t), \mathscr{H}^{n, m}(\psi, x, t)=$ $\mathscr{H}^{\gamma^{n, m}}(\psi, x, t)$. Через $\bar{\rho}^{n, m}(x, t, u)$ обозначим функцию расстояния отображения $\partial_{\psi} \mathscr{H}^{n, m}(0, x, t)$. (Очевидно, что $\mathscr{H}^{n, m}$ есть гамильтониан этого отображения.)

Вернемся к задаче $Z$, в которой $x^{0}(t)$ - сильный минимум. Положим $\eta^{n, m}=$ $c_{0} \eta\left(\gamma^{n, m}\right)$ и рассмотрим задачу $Z^{n, m}$ :

$$
\begin{gathered}
J(p)+\int_{t_{0}}^{t_{1}}\left(x(t)-x^{0}(t)\right)^{2} d t \rightarrow \min , \\
h(p) \leqslant 0, \quad g(p)=0, \\
\dot{x}=u, \quad \bar{\rho}^{n, m}(x, t, u) \leqslant \eta^{n, m} \quad \forall t \in\left[t_{0}, t_{1}\right] .
\end{gathered}
$$

Как показано в п. 3.1, последовательность

$$
\bar{U}^{n, m}(x, t)=\left\{u \mid \bar{\rho}^{n, m}(x, t, u) \leqslant \eta^{n, m}\right\}
$$


приближается сверху к отображению $F(x, t)$. Тогда по теореме 2.1 существует определенная для всех достаточно больших $n, m$ последовательность $x^{n, m}(t)$ сильных минимумов задач $Z^{n, m}$ такая, что $x^{n, m}(t) \rightarrow x^{0}(t)$ при $n \rightarrow \infty, m \rightarrow \infty$ равномерно на $\left[t_{0}, t_{1}\right]$. Для простоты мы будем считать, что эта последовательность определена для всех $n, m$.

Задача $Z^{n, m}$ является задачей оптимального управления с регулярньми смешанными ограничениями в смысле Дубовицкого-Милютина [1]. Более того, она близка к вариационному исчислению и может быть приведена к нему известным приемом Валентайна. Как бы то ни было, но $x^{n, m}(t)$ должно удовлетворять принципу максимума.

Обозначим для краткости $\sigma=(x, t, u)$. Таким образом, $\left(x^{n, m}(t), t, u^{n, m}(t)\right)=$ $\sigma^{n, m}(t)$.

4.2. Формулировка принципа максимума [1]. Существуют числа $\alpha_{0}^{n, m}$, $\beta_{0}^{n, m}, \beta_{1}^{n, m}$, векторы $\alpha^{n, m} \in \mathbb{R}^{d(h)}, \beta^{n, m} \in \mathbb{R}^{d(g)}$, измеримая на $\left[t_{0}, t_{1}\right]$ суммируемая скалярная функция $\lambda^{n, m}(t)$, абсолютно непрерывные функции $\psi^{n, m}(t):\left[t_{0}, t_{1}\right]$ $\rightarrow \mathbb{R}^{d(x)}$ и $\psi_{t}^{n, m}(t):\left[t_{0}, t_{1}\right] \rightarrow \mathbb{R}$ (в обозначении $\psi_{t}^{n, m}$ буква $t$ является нижним индексом, а не переменной, по которой берется производная) такие, что:

а) $\alpha_{0}^{n, m} \geqslant 0, \alpha^{n, m} \geqslant 0, \alpha^{n, m} h\left(p^{n, m}\right)=0$, где $p^{n, m}=\left(x^{n, m}\left(t_{0}\right), x^{n, m}\left(t_{1}\right)\right)$;

$$
\alpha_{0}^{n, m}+\left|\alpha^{n, m}\right|+\left|\beta^{n, m}\right|+\left|\beta_{0}^{n, m}\right|+\left|\beta_{1}^{n, m}\right|=1
$$

b) $\lambda^{n, m}(t) \geqslant 0$, если $t \in \mathscr{E}^{n, m}$, и $\lambda^{n, m}(t)=0$, если $t \notin \mathscr{E}^{n, m}$, где $\mathscr{E} n, m=$ $\left\{t \mid \bar{\rho}^{n, m}\left(\sigma^{n, m}(t)\right)=\eta^{n, m}\right\}$;

c)

$$
\begin{gathered}
\psi^{n, m}(t)= \begin{cases}\lambda^{n, m}(t) \bar{\rho}_{u}^{n, m}\left(\sigma^{n, m}(t)\right), & t \in \mathscr{E} n, m, \\
0, & t \notin \mathscr{E} n, m,\end{cases} \\
\psi^{n, m}(t) u^{n, m}(t)+\psi_{t}^{n, m}(t)-\alpha_{0}^{n, m}\left(x^{n, m}(t)-x^{0}(t)\right)^{2}=0
\end{gathered}
$$

(условия максимума);

d)

$$
\begin{aligned}
& \frac{d}{d t} \psi^{n, m}= \begin{cases}\lambda^{n, m}(t) \bar{\rho}_{x}^{n, m}\left(\sigma^{n, m}(t)\right)+2 \alpha_{0}^{n, m}\left(x^{n, m}(t)-x^{0}(t)\right), & t \in \mathscr{E} n, m, \\
2 \alpha_{0}^{n, m}\left(x^{n, m}(t)-x^{0}(t)\right), & t \notin \mathscr{E} n, m,\end{cases} \\
& \frac{d}{d t} \psi_{t}^{n, m}= \begin{cases}\lambda^{n, m}(t) \bar{\rho}_{t}^{n, m}\left(\sigma^{n, m}(t)\right)-2 \alpha_{0}^{n, m}\left(x^{n, m}(t)-x^{0}(t)\right) \dot{x}^{0}(t), & t \in \mathscr{E} n, m, \\
-2 \alpha_{0}^{n, m}\left(x^{n, m}(t)-x^{0}(t)\right) \dot{x}^{0}(t), & t \notin \mathscr{E} n, m\end{cases}
\end{aligned}
$$

(сопряженные уравнения);

e) $\psi^{n, m}\left(t_{0}\right)=l_{x_{0}}^{n, m}\left(p^{n, m}\right), \psi^{n, m}\left(t_{1}\right)=-l_{x_{1}}^{n, m}\left(p^{n, m}\right)$,

$$
\psi_{t}^{n, m}\left(t_{0}\right)=\beta_{0}^{n, m}, \quad \psi_{t}^{n, m}\left(t_{1}\right)=\beta_{1}^{n, m},
$$

где $l^{n, m}(p)=\alpha_{0}^{n, m} J(p)+\alpha^{n, m} h(p)+\beta^{n, m} g(p)$

(условия трансверсальности).

Таковы условия принципа максимума, полученные Дубовицким и Милютиньм и опубликованные в [1]. 


\section{§5. Анализ принципа максимума}

Для удобства записи обозначим пару $(n, m)$ через $q$, а пару $(\psi, x)$ через $w$.

5.1. Анализ условия максимума (4.1c). На множестве $\mathscr{E} q$ имеем $\bar{\rho}^{q}\left(\sigma^{q}(t)\right)$ $=\eta^{q}>0$. Следовательно, $\left|\bar{\rho}_{u}^{q}\left(\sigma^{q}(t)\right)\right|=1$ на $\mathscr{E} q$. Тогда из (4.1c) следует

$$
\left|\psi^{q}(t)\right|=\lambda^{q}(t), \quad t \in\left[t_{0}, t_{1}\right] .
$$

Покажем далее, что

$$
\psi^{q}(t) u^{q}(t)=\left|\psi^{q}(t)\right| \eta^{q}+\mathscr{H}^{q}\left(w^{q}(t), t\right) .
$$

Очевидно, достаточно доказать выполнение этого равенства на $\mathscr{E} q$. Тогда согласно (4.1c) и формуле двойственности (3.3) с учетом однородности $\mathscr{H}^{q}$ по переменной $\psi$ имеем

$$
\begin{aligned}
\psi^{q}(t) u^{q}(t) & =\lambda^{q}(t) \bar{\rho}_{u}^{q}\left(\sigma^{q}(t)\right) u^{q}(t) \\
& =\left|\psi^{q}(t)\right|\left(\bar{\rho}^{q}\left(\sigma^{q}(t)\right)+\mathscr{H}^{q}\left(\lambda^{q}(t) \bar{\rho}_{u}^{q}\left(\sigma^{q}(t)\right), x^{q}(t), t\right)\right) \\
& =\left|\psi^{q}(t)\right| \eta^{q}+\mathscr{H}^{q}\left(\psi^{q}(t), x^{q}(t), t\right) .
\end{aligned}
$$

Тем самым (5.2) доказано.

Далее, согласно формуле двойственности $\left(3.2^{\prime}\right)$ имеем:

$$
\begin{aligned}
\left(\psi^{q}+\bar{\psi}\right) u^{q}(t) & \leqslant\left|\psi^{q}+\bar{\psi}\right| \bar{\rho}^{q}\left(\sigma^{q}(t)\right)+\mathscr{H}^{q}\left(\psi^{q}(t)+\bar{\psi}, x^{q}(t), t\right) \\
& \leqslant\left|\psi^{q}+\bar{\psi}\right| \eta^{q}+\mathscr{H}^{q}\left(\psi^{q}+\bar{\psi}, x^{q}, t\right)
\end{aligned}
$$

для любого $\bar{\psi}$. Отсюда и из $(5.2)$ в силу сублинейности $\mathscr{H}^{q}$ по $\psi$ легко следует, что почти всюду на $\left[t_{0}, t_{1}\right]$ выполнено неравенство

$$
\bar{\psi} u^{q}(t) \leqslant|\bar{\psi}| \eta^{q}+\mathscr{H}_{\psi}^{q}\left(w^{q}(t), t, \bar{\psi}\right) \quad \forall \bar{\psi} .
$$

5.2. Основное неравенство. Сопряженные уравнения можно записать в виде:

$$
\begin{aligned}
\dot{\psi}^{q}(t) & =-\mathscr{H}_{x}^{q}\left(w^{q}(t), t\right)+2 \alpha_{0}^{q}\left(x^{q}(t)-\widehat{x}^{0}(t)\right), \\
\dot{\psi}_{t}^{q}(t) & =-\mathscr{H}_{t}^{q}\left(w^{q}(t), t\right)-2 \alpha_{0}^{q}\left(x^{q}(t)-\widehat{x}^{0}(t)\right) \dot{x}^{0}(t) .
\end{aligned}
$$

Действительно, достаточно установить выполнение этих уравнений на $\mathscr{E} q$. Но на $\mathscr{E}^{q}$ функция $\bar{\rho}^{q}\left(\sigma^{q}(t)\right)$ положительна, и в силу (3.4) и (3.5) имеем:

$$
\begin{aligned}
& \bar{\rho}_{x}^{q}\left(\sigma^{q}(t)\right)=-\mathscr{H}_{x}^{q}\left(\bar{\rho}_{u}^{q}\left(\sigma^{q}(t)\right), x^{q}(t), t\right), \\
& \bar{\rho}_{t}^{q}\left(\sigma^{q}(t)\right)=-\mathscr{H}_{t}^{q}\left(\bar{\rho}_{u}^{q}\left(\sigma^{q}(t)\right), x^{q}(t), t\right) .
\end{aligned}
$$

Учитывая (5.1), получаем отсюда на $\mathscr{E} q$ :

$$
\begin{aligned}
& \dot{\psi}^{q}(t)=-\left|\psi^{q}(t)\right| \mathscr{H}_{x}^{q}\left(\bar{\rho}_{u}^{q}\left(\sigma^{q}(t)\right), x^{q}(t), t\right)+\alpha_{0}^{q}\left(x^{q}(t)-\widehat{x}^{0}(t)\right), \\
& \dot{\psi}_{t}^{q}(t)=-\left|\psi^{q}(t)\right| \mathscr{H}_{t}^{q}\left(\bar{\rho}_{u}^{q}\left(\sigma^{q}(t)\right), x^{q}(t), t\right)-\alpha_{0}^{q}\left(x^{q}(t)-\widehat{x}^{0}(t)\right) \dot{x}^{0}(t) .
\end{aligned}
$$


В силу однородности $\mathscr{H}^{q}$ по $\psi$ из (4.1c) вытекает, что на $\mathscr{E} q$ имеет место (5.4).

Положим

$$
\eta_{1}^{q}=\max _{\left[t_{0}, t_{1}\right]} 2 \alpha_{0}^{q}\left|x^{q}(t)-x^{0}(t)\right|, \quad \eta_{2}^{q}=\underset{\left[t_{0}, t_{1}\right]}{\operatorname{vrai} \max } 2 \alpha_{0}^{q}\left|x^{q}(t)-x^{0}(t)\right|\left|\dot{x}^{0}(t)\right| .
$$

Тогда из (5.4) следует

$$
-\bar{x} \dot{\psi}^{q}(t)-\bar{t} \dot{\psi}_{t}^{q} \leqslant \mathscr{H}_{x}^{q}\left(w^{q}(t), t\right) \bar{x}+\mathscr{H}_{t}^{q}\left(w^{q}(t), t\right) \bar{t}+\eta_{1}^{q}|\bar{x}|+\eta_{2}^{q}|\bar{t}| \quad \forall \bar{x}, \bar{t}
$$

для почти всех $t \in\left[t_{0}, t_{1}\right]$. Складывая это неравенство с (5.3), получаем следующее основное неравенство: почти всюду на $\left[t_{0}, t_{1}\right]$ выполнено:

$$
\begin{gathered}
\bar{\psi} u^{q}(t)-\bar{x} \dot{\psi}^{q}(t)-\bar{t} \dot{\psi}_{t}^{q} \leqslant \\
\mathscr{H}_{\psi}^{q}\left(w^{q}(t), t ; \bar{\psi}\right)+\mathscr{H}_{x}^{q}\left(w^{q}(t), t\right) \bar{x}+\mathscr{H}_{t}^{q}\left(w^{q}(t), t\right) \bar{t} \\
+\eta^{q}|\bar{\psi}|+\eta_{1}^{q}|\bar{x}|+\eta_{2}^{q}|\bar{t}| \quad \forall \bar{\psi}, \bar{x}, \bar{t}
\end{gathered}
$$

5.3. Равномерная ограниченность. Будем писать $q \rightarrow \infty$, если $n \rightarrow \infty$, $m \rightarrow \infty$. Мы покажем, что последовательности $\psi^{q}(t), \psi_{t}^{q}(t), \dot{\psi}^{q}(t), \dot{\psi}_{t}^{q}(t)$ равномерно ограничены при $q \rightarrow \infty$.

Полагая в $(5.5) \bar{\psi}=0$, получаем

$$
\left|\dot{\psi}^{q}(t)\right| \leqslant\left|\mathscr{H}_{x}^{q}\left(w^{q}, t\right)\right|+\eta_{1}^{q}, \quad\left|\dot{\psi}_{t}^{q}(t)\right| \leqslant\left|\mathscr{H}_{t}^{q}\left(w^{q}, t\right)\right|+\eta_{2}^{q}
$$

Поскольку $\mathscr{H}(w, t)$ удовлетворяет условию Липшица с постоянной $c_{0}$, то

$$
\left|\mathscr{H}_{x}^{q}\left(w^{q}, t\right)\right| \leqslant c_{0}\left|\psi^{q}(t)\right|, \quad\left|\mathscr{H}_{t}^{q}\left(w^{q}, t\right)\right| \leqslant c_{0}\left|\psi^{q}(t)\right|
$$

следовательно,

$$
\left|\dot{\psi}^{q}(t)\right| \leqslant c_{0}\left|\psi^{q}(t)\right|+\eta_{1}^{q}, \quad\left|\dot{\psi}_{t}^{q}(t)\right| \leqslant c_{0}\left|\psi^{q}(t)\right|+\eta_{2}^{q}
$$

Согласно условию трансверсальности (4.1е) и условию нормировки (4.1a) последовательности $\psi^{q}\left(t_{0}\right)$ и $\psi_{t}^{q}\left(t_{0}\right)$ ограничены при $q \rightarrow \infty$.

Кроме того, $\eta_{1}^{q} \rightarrow 0$ и $\eta_{2}^{q} \rightarrow 0$ при $q \rightarrow \infty$, и из (5.6) следует равномерная ограниченность последовательностей $\psi^{q}(t), \dot{\psi}^{q}(t), \dot{\psi}_{t}^{q}(t)$ при $q \rightarrow \infty$. Тогда последовательность $\psi_{t}^{q}(t)$ также равномерно ограничена.

5.4. Выбор подпоследовательностей. В этом пункте нам будет удобнее вновь рассматривать индекс $q$ как пару $n, m$. Из утверждения предыдущего пункта следует, что для всех достаточно больших $n$ и $m$ множество функций $\psi^{q}(t), \psi_{t}^{q}(t)$, $\dot{\psi}^{q}(t), \dot{\psi}_{t}^{q}(t)$ ограничено в $L_{\infty}\left[t_{0}, t_{1}\right]$. Для всех достаточно больших $n$ определим последовательность $\pi^{n}=\left\{m_{1}, \ldots, m_{k}, \ldots\right\}$ (в действительности $m_{k}=m_{k}(n)$ ) такую, что $m_{k} \rightarrow \infty$ (при $\left.k \rightarrow \infty\right)$ и на отрезке $\left[t_{0}, t_{1}\right]$ существуют пределы

$$
\lim _{m \in \pi^{n}} x^{n, m}(t)=x^{n}(t), \quad \lim _{m \in \pi^{n}} \psi^{n, m}(t)=\psi^{n}(t), \quad \lim _{m \in \pi^{n}} \psi_{t}^{n, m}(t)=\psi_{t}^{n}(t)
$$


Очевидно, что множество функций

$$
\dot{x}^{n}(t)=u^{n}(t), \quad \psi^{n}(t), \quad \psi_{t}^{n}(t), \quad \dot{\psi}^{n}(t), \quad \dot{\psi}_{t}^{n}(t)
$$

ограничено в $L_{\infty}$. Выберем последовательность $\pi=\left\{n_{1}, n_{2}, \ldots\right\}$ так, чтобы $n_{k} \rightarrow \infty($ при $k \rightarrow \infty)$ и на отрезке $\left[t_{0}, t_{1}\right]$ сушествовали пределы

$$
\lim _{n \in \pi} \psi^{n}(t)=\psi^{0}(t), \quad \lim _{n \in \pi} \psi_{t}^{n}(t)=\psi_{t}^{0}(t)
$$

Ясно, что при этом $\lim _{n \in \pi} x^{n}(t)=x^{0}(t)$.

Наконец, для $n \in \pi$ определим $q(n)$ из условий: $q(n)=(n, m)$, где $m=m_{k_{n}}(n) \in$ $\pi^{n}$, и

$$
\max _{\left[t_{0}, t_{1}\right]}\left|\psi^{n}(t)-\psi^{q(n)}(t)\right| \rightarrow 0, \quad \max _{\left[t_{0}, t_{1}\right]}\left|\psi_{t}^{n}(t)-\psi_{t}^{q(n)}(t)\right| \rightarrow 0
$$

при $n \rightarrow \infty, n \in \pi$. Очевидно, при $n \rightarrow \infty, n \in \pi$ имеем

$$
\psi^{q(n)}(t) \rightarrow \psi^{0}(t), \quad \psi_{t}^{q(n)}(t) \rightarrow \psi_{t}^{0}(t)
$$

Не ограничивая общности, считаем также, что $\left(\alpha_{0}^{q(n)}, \alpha^{q(n)}, \beta^{q(n)}\right) \rightarrow\left(\alpha_{0}^{0}, \alpha^{0}, \beta^{0}\right)$.

Итак, выбор подпоследовательностей сделан.

\section{§6. Необходимые условия}

6.1. Предельный переход по последовательности $\{q(n)\}, n \in \pi$. При $n \rightarrow \infty, n \in \pi$ имеем

$$
u^{q(n)}(t) \rightarrow u^{0}(t)=\dot{x}^{0}(t), \quad \eta^{q(n)} \rightarrow 0, \quad \mathscr{H}^{q}\left(w^{q}, t\right) \rightarrow \mathscr{H}\left(w^{0}, t\right)
$$

Тогда из (5.2) следует, что

$$
\psi^{0}(t) \dot{x}^{0}(t)=\mathscr{H}\left(w^{0}(t), t\right)
$$

Переходя к пределу во втором условии (4.1c) и учитывая (6.1), получаем

$$
\mathscr{H}\left(w^{0}(t), t\right)+\psi_{t}^{0}(t)=0
$$

Докажем, что тогда почти всюду на $\left[t_{0}, t_{1}\right]$ выполнено неравенство

$$
\bar{\psi} \dot{x}^{0}(t)-\bar{x} \dot{\psi}^{0}(t)-\bar{t} \dot{\psi}_{t}^{0}(t) \leqslant \stackrel{\circ}{\mathscr{H}}_{\mathrm{Cl}}\left(w^{0}(t), t ; \bar{\psi}, \bar{x}, \bar{t}\right) \quad \forall \bar{\psi}, \bar{x}, \bar{t}
$$

где $\stackrel{\circ}{\mathscr{H}_{\mathrm{Cl}}}\left(w^{0}(t), t ; \bar{\psi}, \bar{x}, \bar{t}\right)$ - производная Кларка в точке $\left(w^{0}(t), t\right)$ по направлению $(\bar{\psi}, \bar{x}, \bar{t})$.

Действительно, так как $\mathscr{H}^{q}$ обладает непрерывной производной по $x, t$ и сублинейна по $\psi$, то

$$
\mathscr{H}_{\psi}^{q}\left(w^{q}(t), t ; \bar{\psi}\right)+\mathscr{H}_{x}^{q}\left(w^{q}(t), t\right) \bar{x}+\mathscr{H}_{t}^{q}\left(w^{q}(t), t\right) \bar{t}=\stackrel{\circ}{\mathscr{H}}^{q}\left(w^{q}(t), t ; \bar{\psi}, \bar{x}, \bar{t}\right)
$$


- производная функции $\mathscr{H}^{q}$ в точке $\left(w^{q}(t), t\right)$ по направлению $(\bar{\psi}, \bar{x}, \bar{t})$.

Применяя к основному неравенству (5.5) лемму 1.1 , получаем почти всюду на $\left[t_{0}, t_{1}\right]:$

$$
\bar{\psi} \dot{x}^{0}(t)-\bar{x} \dot{\psi}^{0}(t)-\bar{t} \dot{\psi}_{t}^{0}(t) \leqslant \varlimsup_{n \in \pi} \stackrel{\circ}{\mathscr{H}}^{q(n)}\left(w^{q(n)}(t), t ; \bar{\psi}, \bar{x}, \bar{t}\right) \quad \forall \bar{\psi}, \bar{x}, \bar{t}
$$

Но, как нетрудно видеть,

$$
\stackrel{\circ}{\mathscr{H}}^{q}(\psi, x, t ; \bar{\psi}, \bar{x}, \bar{t}) \leqslant \int d x^{\prime} d t^{\prime}\left\{\stackrel{\circ}{\mathscr{H}_{\text {вер }}}\left(\psi, x^{\prime}, t^{\prime} ; \bar{\psi}, \bar{x}, \bar{t}\right) \gamma^{q}\left(x^{\prime}-x, t^{\prime}-t\right)\right\}
$$

где $\stackrel{\circ}{\mathscr{H}_{\text {вер }}}$ - верхняя производная по направлению $\bar{\psi}, \bar{x}, \bar{t}$. Подставляя эту оценку в (6.4), легко получаем (6.3).

Наконец, покажем, что

$$
\alpha_{0}^{0}+\left|\alpha^{0}\right|+\left|\beta^{0}\right|>0
$$

Действительно, допустим противное. Согласно условию трансверсальности $\psi^{0}\left(t_{0}\right)=l_{x_{0}}^{0}\left(p^{0}\right)$, где $l^{0}(p)=\alpha_{0}^{0} J(p)+\alpha^{0} h(p)+\beta^{0} g(p)$, получим $\psi^{0}\left(t_{0}\right)=0$. Тогда из (6.3) следует $\psi^{0}(t)=0 \forall t$. Согласно (6.2) получим отсюда, что $\psi_{t}^{0}(t)=0$ $\forall t$. Но это противоречит условию нормировки (4.1) для предельных величин. Тем самьм свойство (6.5) доказано.

6.2. Последовательный переход к пределу. Для $n \in \pi$ оценим сверху величину

$$
A^{n}=\varlimsup_{m \in \pi^{n}}\left(\mathscr{H}_{\psi}^{n, m}\left(w^{n, m}(t), t ; \bar{\psi}\right)+\mathscr{H}_{x}^{n, m}\left(w^{n, m}(t), t\right) \bar{x}+\mathscr{H}_{t}^{n, m}\left(w^{n, m}(t), t\right) \bar{t}\right) .
$$

Очевидно, $A^{n} \leqslant A_{\psi}^{n}+A_{x}^{n}+A_{t}^{n}$, где

$$
\begin{aligned}
A_{\psi}^{n} & =\varlimsup_{m \in \pi^{n}} \mathscr{H}_{\psi}^{n, m}\left(w^{n, m}(t), t ; \bar{\psi}\right), \\
A_{x}^{n} & =\varlimsup_{m \in \pi^{n}} \mathscr{H}_{x}^{n, m}\left(w^{n, m}(t), t\right) \bar{x} \\
A_{t}^{n} & =\varlimsup_{m \in \pi^{n}} \mathscr{H}_{t}^{n, m}\left(w^{n, m}(t), t\right) \bar{t}
\end{aligned}
$$

Ясно, что $\mathscr{H}^{n, m}\left(\psi^{n, m}(t)+\tau \bar{\psi}, x^{n, m}(t), t\right)$ - выпуклая функция по $\tau$, сходящаяся при $m \rightarrow \infty, m \in \pi^{n}$ к $\mathscr{H}^{n}\left(\psi^{n}(t)+\tau \bar{\psi}, x^{n}(t), t\right), \tau \in[0,1]$, где $\mathscr{H}^{n}(w, t)=$ $\mathscr{H}^{\mu^{n}}(w, t)$. Отсюда следует

$$
A_{\psi}^{n} \leqslant \mathscr{H}_{\psi}^{n}\left(w^{n}(t), t ; \bar{\psi}\right)
$$

Далее, имеем: $\mathscr{H}_{x}^{n, m}(w, t) \rightarrow \mathscr{H}_{x}^{n}(w, t)$ равномерно на каждом ограниченном множестве $w, t$. Отсюда $\mathscr{H}_{x}^{n, m}\left(w^{n, m}(t), t\right) \rightarrow \mathscr{H}_{x}^{n}\left(w^{n}(t), t\right)$ при $m \rightarrow \infty, m \in \pi^{n}$. Следовательно,

$$
A_{x}^{n}=\mathscr{H}_{x}^{n}\left(w^{n}(t), t\right) \bar{x}
$$


Наконец, $A_{t}^{n}$ представим в виде

$$
A_{t}^{n}=\varlimsup_{m \in \pi^{n}} \int d x^{\prime}\left\{\mathscr{H}_{t}^{\nu^{m}}\left(\psi^{n, m}(t), x^{\prime}, t\right) \bar{t} \mu^{n}\left(x^{\prime}-x^{n, m}(t)\right)\right\} .
$$

Положим

$$
\Phi(\psi, x, t ; \bar{t})=\varlimsup\left\{\mathscr{H}_{t}^{\nu^{m}}\left(\psi^{\prime}, x, t\right) \bar{t} \mid \psi^{\prime} \rightarrow \psi, m \rightarrow \infty\right\}
$$

и обозначим $\Phi^{n}=\Phi^{\mu^{n}}$. Тогда из представления (6.8) получим

$$
A_{t}^{n} \leqslant \Phi^{n}\left(w^{n}(t), t ; \bar{t}\right)
$$

Переходя в (5.5) к пределу по $m \rightarrow \infty, m \in \pi^{n}$, получаем согласно (6.6), (6.7), $(6.9)$ :

$$
\begin{gathered}
\bar{\psi} u^{n}(t)-\bar{x} \dot{\psi}^{n}(t)-\bar{t} \dot{\psi}_{t}^{n}(t) \leqslant \\
\mathscr{H}_{\psi}^{n}\left(w^{n}(t), t ; \bar{\psi}\right)+\mathscr{H}_{x}^{n}\left(w^{n}(t), t\right) \bar{x}+\Phi^{n}\left(w^{n}(t), t ; \bar{t}\right) \\
+\eta^{n}|\bar{\psi}|+\eta_{1}^{n}|\bar{x}|+\eta_{2}^{n}|\bar{t}| \quad \forall \bar{\psi}, \bar{x}, \bar{t}
\end{gathered}
$$

где $\eta^{n}=\varlimsup_{m \in \pi^{n}} \eta^{n, m}, \eta_{1}^{n}=\varlimsup_{m \in \pi^{n}} \eta_{1}^{n, m}, \eta_{2}^{n}=\varlimsup_{m \in \pi^{n}} \eta_{2}^{n, m}$.

Неравенство (6.10) имеет место для почти всех $t \in\left[t_{0}, t_{1}\right]$.

При фиксированном $t$ рассмотрим функцию $\mathscr{H}[t](\psi, x)=\mathscr{H}(\psi, x ; t)$. Очевидно,

$$
\begin{aligned}
\mathscr{H}_{\psi}^{n}\left(w^{n}(t), t ; \bar{\psi}\right) & =(\mathscr{H}[t])_{\psi}^{n}\left(w^{n}(t) ; \bar{\psi}\right), \\
\mathscr{H}_{x}^{n}\left(w^{n}(t), t\right) \bar{x} & =(\mathscr{H}[t])_{x}^{n}\left(w^{n}(t)\right) \bar{x}
\end{aligned}
$$

Так как функция $(\mathscr{H}[t])_{x}^{n}(w)$ непрерывна по $w$, то сумму первых двух членов в правой части (6.10) можно представить в виде $(\stackrel{\circ}{\mathscr{H}}[t])^{n}\left(w^{n}(t) ; \bar{\psi}, \bar{x}\right)$. Устремляя $n$ к бесконечности, $n \in \pi$, и оценивая сверху средние значения, получаем

$$
\begin{aligned}
& \bar{\psi} \dot{x}^{0}(t)-\bar{x} \dot{\psi}^{0}(t)-\bar{t} \dot{\psi}_{t}^{0}(t) \\
& \quad \leqslant \overline{\lim }\left\{(\stackrel{\circ}{\mathscr{H}}[t])_{\text {вер }}\left(w^{\prime} ; \bar{\psi}, \bar{x}\right)+\Phi\left(w^{\prime}, t ; \bar{t}\right) \mid w^{\prime} \rightarrow w^{0}(t)\right\} \quad \forall \bar{\psi}, \bar{x}, \bar{t} .
\end{aligned}
$$

Это неравенство справедливо на множестве полной меры.

Собирая полученные условия, сформулируем результат.

ТЕОрема 6.1. Если $x^{0}(t)$ - сильный минимум задачи $Z$, то для любой последовательности $\nu^{m}(t)$ такой, что $\eta\left(\nu^{m}\right) \rightarrow 0$ при $m \rightarrow \infty$, существуют $\alpha_{0}^{0}, \alpha^{0}, \beta^{0}$ и функиии $\psi^{0}(t), \psi_{t}^{0}(t)$ такие, что

a)

$$
\alpha_{0}^{0} \geqslant 0, \quad \alpha^{0} \geqslant 0, \quad \alpha_{0}^{0}+\left|\alpha^{0}\right|+\left|\beta^{0}\right|>0, \quad\left(\alpha^{0}, h\left(p^{0}\right)\right)=0
$$

b)

$$
\left(\psi\left(t_{0}\right),-\psi\left(t_{1}\right)\right)=l_{p}^{0}\left(p^{0}\right)
$$

c)

$$
\psi^{0}(t) \dot{x}^{0}(t)=\mathscr{H}\left(w^{0}(t), t\right)=-\psi_{t}^{0}(t)
$$


$\left.\mathrm{d}^{\prime}\right)$ на множсестве полной мерь на $\left[t_{0}, t_{1}\right]$ справедливо неравенство

$$
\bar{\psi} \dot{x}^{0}(t)-\bar{x} \dot{\psi}^{0}(t)-\bar{t} \dot{\psi}_{t}^{0}(t) \leqslant \stackrel{\circ}{\mathscr{H}}_{\mathrm{Cl}}\left(w^{0}(t), t ; \bar{\psi}, \bar{x}, \bar{t}\right) \quad \forall \bar{\psi}, \bar{x}, \bar{t}
$$

$\left.\mathrm{d}^{\prime \prime}\right)$ на множсестве полной меры справедливо неравенство

$$
\begin{aligned}
\bar{\psi} \dot{x}^{0}(t) & -\bar{x} \dot{\psi}^{0}(t)-\bar{t} \dot{\psi}_{t}^{0}(t) \\
\leqslant & \left\lceil\stackrel{\circ}{\lim }\left\{\stackrel{\mathscr{H}}{ }[t]_{\mathrm{вер}}\left(w^{\prime} ; \bar{\psi}, \bar{x}\right)+\Phi\left(w^{\prime}, t ; \bar{t}\right) \mid w^{\prime} \rightarrow w^{0}(t)\right\} \quad \forall \bar{\psi}, \bar{x}, \bar{t},\left(6.12 \mathrm{~d}^{\prime \prime}\right)\right.
\end{aligned}
$$

где функиия $\Phi$ определена равенством $\left(6.7^{\prime}\right)$ и зависит от последовательнос$m u\left\{\nu^{m}\right\}$.

Условия а), b), c), d' ) - это условия Кларка [2]. Условие $\mathrm{d}^{\prime \prime}$ ) - новое дополнение к ним. Ниже мы увидим, что оно не следует из условий Кларка.

Отметим, что в $\left(6.12 \mathrm{~d}^{\prime \prime}\right)$ компонента $x^{\prime}$ может принадлежать (для почти всех $t$ ) некоему множеству полной меры по $x$. Это вытекает из неравенства (6.10). Мы воспользуемся этим в дальнейшем. Таковы необходимые условия.

В $\S 7$ мы проведем анализ условия $\left(6.12 \mathrm{~d}^{\prime \prime}\right)$ для семейства $\left\{\nu^{\delta}(t)\right\}_{\delta \rightarrow 0}$ вида

$$
\begin{aligned}
& \nu^{\delta}(t)=\frac{1}{\delta} \chi_{[0, \delta]}(t), \quad \text { если } \delta>0, \\
& \nu^{\delta}(t)=-\frac{1}{\delta} \chi_{[\delta, 0]}(t), \text { если } \delta<0,
\end{aligned}
$$

где $\chi_{I}(t)$ есть характеристическая функция отрезка $I$. Для этого семейства, очевидно, имеем

$$
\Phi(\psi, x, t ; \bar{t})=\varlimsup\left\{\frac{\mathscr{H}\left(\psi^{\prime}, x, t+\delta\right)-\mathscr{H}\left(\psi^{\prime}, x, t\right)}{\delta} \cdot \bar{t} \mid \psi^{\prime} \rightarrow \psi, \delta \rightarrow 0\right\}
$$

\section{§7. Примеры}

Мы рассмотрим гамильтониан вида:

$$
\mathscr{H}(\psi, x, t)=\sum_{k=1}^{d(\varphi)} \lambda_{k}(x, t) \varphi_{k}\left(\psi f_{k}(x, t)\right)
$$

где при каждом $k \in\{1, \ldots, d(\varphi)\} \varphi_{k}:\left(\mathbb{R}^{m_{k}}\right)^{*} \rightarrow \mathbb{R}-$ сублинейный функционал, $\psi=\left(\psi_{1}, \ldots, \psi_{d(x)}\right) \in\left(\mathbb{R}^{d(x)}\right)^{*}$ - вектор-строка размерности $d(x), f_{k}(x, t)$ - $\left(d(x) \times m_{k}\right)$-матрица, зависящая от $(x, t), \lambda_{k}(x, t)$ - неотрицательная скалярная функция, причем предполагается, что функции $\lambda_{k}(x, t)$ и $f_{k}(x, t)$ удовлетворяют условию Липшица по $(x, t)$. Положим $\lambda(x, t)=\left(\lambda_{1}(x, t), \ldots, \lambda_{d(\varphi)}(x, t)\right), \varphi=$ $\left(\varphi_{1}, \ldots, \varphi_{d(\varphi)}\right)$, а через $f(x, t)$ обозначим набор матриц $f_{k}(x, t), k=1, \ldots, d(\varphi)$. Гамильтониан кратко запишем в виде

$$
\mathscr{H}(\psi, x, t)=\lambda(x, t) \varphi(\psi f(x, t))
$$


как если бы $d(\varphi)=1$. Дальнейшие выкладки соответствуют этому случаю (в общем случае следует проводить суммирование соответствующих членов по $k=$ $1, \ldots, d(\varphi))$.

Пусть $\Sigma$ есть множество $(x, t)$, для которых существуют полные дифференциалы $\lambda^{\prime}(x, t), f^{\prime}(x, t)$. Пусть точка $(\psi, x, t)$ такова, что $(x, t) \in \Sigma$. Тогда, как нетрудно видеть,

$$
\stackrel{\circ}{\mathscr{H}}(\varphi, x, t ; \bar{\varphi}, \bar{x}, \bar{t})=\bar{\lambda}(x, t) \varphi(\psi f(x, t))+\lambda(x, t) \stackrel{\circ}{\varphi}(\psi f(x, t) ; \bar{\psi} f(x, t)+\psi \bar{f}(x, t)),
$$

где $\bar{\lambda}(x, t)=\lambda_{x}(x, t) \bar{x}+\lambda_{t}(x, t) \bar{t}, \bar{f}(x, t)=f_{x}(x, t) \bar{x}+f_{t}(x, t) \bar{t}$.

Вычислим $\Phi(\psi, x, t ; \bar{t})$ для семейства $\left\{\nu^{\delta}(t)\right\}$, введенного в предыдушем параграфе. Нам потребуется следуюшая лемма.

ЛЕмма 7.1. Пусть $(\xi)$ - сублинейный функционал. Пусть имеются последовательности $\xi^{\prime} \rightarrow \xi, \bar{\xi}^{\prime} \rightarrow \bar{\xi}, \tau \rightarrow+0$. Тогда верхний предел

$$
\varlimsup \frac{\zeta\left(\xi^{\prime}+\tau \bar{\xi}^{\prime}\right)-\zeta\left(\xi^{\prime}\right)}{\tau} \leqslant \stackrel{\circ}{\zeta}(\xi, \bar{\xi}) .
$$

Доказательство леммы элементарно, и мы его опускаем.

С помошью этой леммы докажем следуюшее предложение.

ПреДЛОЖЕНИЕ 7.1. Пусть $(x, t) \in \Sigma$. Тогда $\Phi(\psi, x, t ; \bar{t})=\stackrel{\circ}{\mathscr{H}}(\varphi, x, t ; 0,0, \bar{t})$.

ДокАЗАТЕЛЬство. Легко видеть, что

$$
\begin{aligned}
& \Phi(\psi, x, t ; \bar{t})=\max \left\{\varlimsup_{\psi^{\prime} \rightarrow \psi, \delta \rightarrow+0} \frac{\mathscr{H}\left(\psi^{\prime}, x, t+\delta\right)-\mathscr{H}\left(\psi^{\prime}, x, t\right)}{\delta} \bar{t} ;\right. \\
& \left.\varlimsup_{\psi^{\prime} \rightarrow \psi, \delta \rightarrow+0} \frac{\mathscr{H}\left(\psi^{\prime}, x, t\right)-\mathscr{H}\left(\psi^{\prime}, x, t-\delta\right)}{\delta} \bar{t}\right\} .
\end{aligned}
$$

Рассмотрим случай $\bar{t}>0$. Положим

$$
\begin{aligned}
b_{1} & =\varlimsup_{\psi^{\prime} \rightarrow \psi, \delta \rightarrow+0} \frac{\mathscr{H}\left(\psi^{\prime}, x, t+\delta\right)-\mathscr{H}\left(\psi^{\prime}, x, t\right)}{\delta}, \\
b_{2} & =\varlimsup_{\psi^{\prime} \rightarrow \psi, \delta \rightarrow+0} \frac{\mathscr{H}\left(\psi^{\prime}, x, t\right)-\mathscr{H}\left(\psi^{\prime}, x, t-\delta\right)}{\delta} .
\end{aligned}
$$

Вычисление $b_{1}$. Имеем:

$$
\begin{aligned}
& \mathscr{H}\left(\psi^{\prime}, x, t+\delta\right)-\mathscr{H}\left(\psi^{\prime}, x, t\right) \\
& \quad=\lambda(x, t+\delta) \varphi\left(\psi^{\prime} f(x, t+\delta)\right)-\lambda(x, t) \varphi\left(\psi^{\prime} f(x, t)\right) \\
& \quad=\lambda_{t}(x, t) \varphi\left(\psi^{\prime} f(x, t)\right) \delta+\lambda(x, t)\left(\varphi\left(\psi^{\prime} f(x, t)+\psi^{\prime} f_{t}(x, t) \delta\right)-\varphi\left(\psi^{\prime} f(x, t)\right)\right)+o(\delta) .
\end{aligned}
$$

Отсюда согласно лемме 7.1 получаем равенство

$$
b_{1}=\lambda_{t}(x, t) \varphi(\psi f(x, t))+\lambda(x, t) \stackrel{\circ}{\varphi}\left(\psi f(x, t) ; \psi f_{t}(x, t)\right)
$$


Оценка $b_{2}$. Имеем:

$$
\begin{aligned}
& \mathscr{H}\left(\psi^{\prime}, x, t\right)-\mathscr{H}\left(\psi^{\prime}, x, t-\delta\right) \\
& \quad=\lambda(x, t) \varphi\left(\psi^{\prime} f(x, t)\right)-\lambda(x, t-\delta) \varphi\left(\psi^{\prime} f(x, t-\delta)\right) \\
& \quad=\lambda_{t}(x, t) \varphi\left(\psi^{\prime} f(x, t)\right) \delta-\lambda(x, t)\left(\varphi\left(\psi^{\prime} f(x, t)-\psi^{\prime} f_{t}(x, t) \delta\right)-\varphi\left(\psi^{\prime} f(x, t)\right)\right)+o(\delta) \\
& \quad=\lambda_{t}(x, t) \varphi\left(\psi^{\prime} f(x, t)\right) \delta+\lambda(x, t)\left(\varphi\left(\psi^{\prime} f(x, t)\right)-\varphi\left(\psi^{\prime} f(x, t)-\psi^{\prime} f_{t}(x, t) \delta\right)\right)+o(\delta) .
\end{aligned}
$$

Отсюда согласно лемме 7.1 получим $b_{2} \leqslant b_{1}$. Следовательно, для $\bar{t}>0$ имеем:

$$
\begin{aligned}
\Phi(\psi, x, t ; \bar{t}) & =\lambda_{t}(x, t) \bar{t} \cdot \varphi(\psi f(x, t))+\lambda(x, t) \stackrel{\circ}{\varphi}\left(\psi f(x, t) ; \psi f_{t}(x, t) \bar{t}\right) \\
& =\stackrel{\mathscr{H}}{ }(\varphi, x, t ; 0,0, \bar{t}) .
\end{aligned}
$$

Таким образом, утверждение предложения справедливо для $\bar{t}>0$.

Рассмотрим случай $\bar{t}<0$. Положим:

$$
\begin{aligned}
b_{1}^{\prime} & =\varlimsup_{\psi^{\prime} \rightarrow \psi, \delta \rightarrow+0} \frac{\mathscr{H}\left(\psi^{\prime}, x, t\right)-\mathscr{H}\left(\psi^{\prime}, x, t+\delta\right)}{\delta}, \\
b_{2}^{\prime} & =\varlimsup_{\psi^{\prime} \rightarrow \psi, \delta \rightarrow+0} \frac{\mathscr{H}\left(\psi^{\prime}, x, t-\delta\right)-\mathscr{H}\left(\psi^{\prime}, x, t\right)}{\delta} .
\end{aligned}
$$

Очевидно,

$$
\Phi(\psi, x, t ; \bar{t})=|\bar{t}| \cdot \max \left\{b_{1}^{\prime} ; b_{2}^{\prime}\right\}
$$

Вычисление $b_{2}^{\prime}$. Имеем:

$$
\begin{aligned}
& \mathscr{H}\left(\psi^{\prime}, x, t-\delta\right)-\mathscr{H}\left(\psi^{\prime}, x, t\right) \\
& =\lambda(x, t-\delta) \varphi\left(\psi^{\prime} f(x, t-\delta)\right)-\lambda(x, t) \varphi\left(\psi^{\prime} f(x, t)\right) \\
& =-\lambda_{t}(x, t) \varphi\left(\psi^{\prime} f(x, t)\right) \delta+\lambda(x, t)\left(\varphi\left(\psi^{\prime} f(x, t)-\psi^{\prime} f_{t}(x, t) \delta\right)-\varphi\left(\psi^{\prime} f(x, t)\right)\right)+o(\delta) .
\end{aligned}
$$

Отсюда согласно лемме 7.1 имеем:

$$
b_{2}^{\prime}=-\lambda_{t}(x, t) \varphi(\psi f(x, t))+\lambda(x, t) \stackrel{\circ}{\varphi}\left(\psi f(x, t) ;-\psi f_{t}(x, t)\right) .
$$

Оценка $b_{1}^{\prime}$. Имеем:

$$
\begin{aligned}
& \mathscr{H}\left(\psi^{\prime}, x, t\right)-\mathscr{H}\left(\psi^{\prime}, x, t+\delta\right) \\
& =\lambda(x, t) \varphi\left(\psi^{\prime} f(x, t)\right)-\lambda(x, t+\delta) \varphi\left(\psi^{\prime} f(x, t+\delta)\right) \\
& =-\lambda_{t}(x, t) \varphi\left(\psi^{\prime} f(x, t)\right) \delta-\lambda(x, t)\left(\varphi\left(\psi^{\prime} f(x, t)+\psi^{\prime} f_{t}(x, t) \delta\right)-\varphi\left(\psi^{\prime} f(x, t)\right)\right)+o(\delta) \\
& =\lambda_{t}(x, t) \varphi\left(\psi^{\prime} f(x, t)\right) \delta+\lambda(x, t)\left(\varphi\left(\psi^{\prime} f(x, t)\right)-\varphi\left(\psi^{\prime} f(x, t)-\psi^{\prime} f_{t}(x, t) \delta\right)\right) .
\end{aligned}
$$

Отсюда по лемме 7.1 имеем $b_{1}^{\prime} \leqslant b_{2}^{\prime}$. Следовательно,

$$
\begin{aligned}
\Phi(\psi, x, t ; \bar{t}) & =b_{2}^{\prime} \cdot|\bar{t}|=\lambda_{t}(x, t) \bar{t} \varphi(\psi f(x, t))+\lambda(x, t) \stackrel{\circ}{\varphi}\left(\psi f(x, t) ; \psi f_{t}(x, t) \bar{t}\right) \\
& =\stackrel{\circ}{\mathscr{H}}(\varphi, x, t ; 0,0, \bar{t}) .
\end{aligned}
$$


Тем самым предложение 7.1 доказано.

Для гамильтониана рассматриваемого вида правые части в условиях $\left(6.12 \mathrm{~d}^{\prime}\right)$ и $\left(6.12 \mathrm{~d}^{\prime \prime}\right)$ имеют согласно (7.1) и предложению 7.1 следуюший вид: правая часть условия $\left(6.12 \mathrm{~d}^{\prime}\right)-$

$$
\left.\varlimsup \stackrel{\circ}{\lim }\left(\psi^{\prime}, x^{\prime}, t^{\prime} ; \bar{\psi}, \bar{x}, \bar{t}\right) \mid\left(x^{\prime}, t^{\prime}\right) \in \Sigma,\left(\psi^{\prime}, x^{\prime}, t^{\prime}\right) \rightarrow\left(\psi^{0}(t), x^{0}(t), t\right)\right\},
$$

правая часть условия $\left(6.12 \mathrm{~d}^{\prime \prime}\right)-$

$\left.\varlimsup \stackrel{\circ}{\mathscr{H}}\left(\psi^{\prime}, x^{\prime}, t ; \bar{\psi}, \bar{x}, 0\right)+\stackrel{\circ}{\mathscr{H}}\left(\psi^{\prime}, x^{\prime}, t ; 0,0, \bar{t}\right) \mid x^{\prime} \in \Sigma(t),\left(\psi^{\prime}, x^{\prime}\right) \rightarrow\left(\psi^{0}(t), x^{0}(t)\right)\right\}$,

где $\Sigma(t)=\{x \mid(x, t) \in \Sigma\}$ (подчеркнем, что здесь $t$ фиксировано).

Для данных $\psi, x, t$, где $(x, t) \in \Sigma$, имеет место неравенство

$$
\stackrel{\circ}{\mathscr{H}}(\psi, x, t ; \bar{\psi}, \bar{x}, \bar{t}) \leqslant \stackrel{\circ}{\mathscr{H}}(\psi, x, t ; \bar{\psi}, \bar{x}, 0)+\stackrel{\circ}{\mathscr{H}}(\psi, x, t ; 0,0, \bar{t}),
$$

которое следует в нашем случае из сублинейности $\stackrel{\circ}{\mathscr{H}}$ по $(\bar{\psi}, \bar{x}, \bar{t})$. Это неравенство действует “в пользу” условия (6.12d'). Однако последовательности, по которым берется верхний предел, в случае $\left(7.2^{\prime}\right)$ образуют более богатое множество, чем в случае $\left(7.2^{\prime \prime}\right)$, и это обстоятельство действует “в пользу" условия $\left(6.12 \mathrm{~d}^{\prime \prime}\right)$. Поэтому априори указанные два условия несравнимы, и добавление условия $\left(6.12 \mathrm{~d}^{\prime \prime}\right)$ к условию $\left(6.12 \mathrm{~d}^{\prime}\right)$ может уточнить последнее.

Отметим случаи, когда одно из этих условий оказьвается сильнее другого.

$1)$ Если функции $\lambda(x, t)$ и $f(x, t)$ непрерьвно дифференцируемы по $(x, t)$, то правая часть условия $\left(6.12 \mathrm{~d}^{\prime}\right)$ не превосходит правую часть условия $\left(6.12 \mathrm{~d}^{\prime \prime}\right)$.

$2)$ Если для любого $i \in[0, d(\varphi)]$ функционал $\varphi_{i}$ либо линеен, либо $f_{i}$ не зависит от $t$, то правая часть $\left(6.12 \mathrm{~d}^{\prime \prime}\right)$ не превосходит правую часть $\left(6.12 \mathrm{~d}^{\prime}\right)$.

Однако в случае 1$)$ преимущество условия $\left(6.12 \mathrm{~d}^{\prime}\right)$ кажущееся (подчеркнем, что речь идет о рассматриваемом классе гамильтонианов). Дело в том, что условие $\left(6.12 \mathrm{~d}^{\prime}\right)$ в этом случае эквивалентно принципу максимума Понтрягина для траектории $x^{0}(t)$, а условие $\left(6.12 \mathrm{~d}^{\prime \prime}\right)$ не слабее “укороченного" принципа максимума Понтрягина, в котором в отличие от полного принципа максимума отсутствует сопряженное уравнение на функцию Понтрягина $H$, но, как известно, это уравнение следует из условий укороченного принципа максимума.

Значительно больше информации дает нам случай 2). Рассмотрим следующий пример, относящийся к этому случаю. Пусть функция $g(x)$ скалярного переменного $x$ ограничена сверху и снизу положительными постоянными, удовлетворяет условию Липшица, причем $g_{x}(x)= \pm 1$, и удовлетворяет неравенству

$$
\left|g\left(x_{1}\right)-g\left(x_{2}\right)\right|<\left|x_{1}-x_{2}\right| \text { для любых } x_{1} \neq x_{2} \text {. }
$$

Пусть $x^{0}(t)$ есть решение уравнения

$$
\dot{x}^{0}(t)=g\left(x^{0}(t)\right), \quad x^{0}(0)=0 .
$$


Определим класс функций $f(x, t)$. Обозначим через $Q=\left\{(x, t) \mid x>x^{0}(t)\right\}$ открытый надграфик функции $x^{0}(t)$. Положим

$$
f_{0}(x, t)=\min \{|\bar{x}|+|\bar{t}|-1 ; 0\} .
$$

Зададим последовательности $\left\{x_{k}, t_{k}\right\},\left\{\gamma_{k}^{1}, \gamma_{k}^{2}\right\},\left\{\delta_{k}\right\}$ так, чтобы:

$$
\left(x_{k}, t_{k}\right) \in Q, \quad \overline{\operatorname{lt}}\left(x_{k}, t_{k}\right)=\partial Q, \quad \gamma_{k}^{1}, \gamma_{k}^{2}>0, \quad \delta_{k}>0, \quad \sum \frac{\delta_{k}}{\min \left\{\gamma_{k}^{1}, \gamma_{k}^{2}\right\}}<\infty
$$

Положим

$$
\begin{aligned}
& f_{k}(x, t)=\delta_{k} f_{0}\left(\frac{\gamma_{k}^{1}\left(x-x_{k}\right)}{\delta_{k}}, \frac{\gamma_{k}^{2}\left(t-t_{k}\right)}{\delta_{k}}\right), \\
& D_{k}=\left\{(x, t)\left|\gamma_{k}^{1}\right| x-x_{k}\left|+\gamma_{k}^{2}\right| t-t_{k} \mid \leqslant \delta_{k}\right\} .
\end{aligned}
$$

Потребуем, чтобы $D_{k} \subset Q \forall k$ и $D_{k_{1}} \cap D_{k_{2}}=\varnothing$ при $k_{1} \neq k_{2}$.

Положим $f(x, t)=\sum_{1}^{\infty} f_{k}(x, t)$. Фиксируем отрезок $\left[t_{0}, t_{1}\right]$. Очевидно, $f\left(x^{0}(t), t\right)=0$, поэтому $x^{0}(t)$ удовлетворяет уравнению

$$
\dot{x}=f(x, t)+g(x)
$$

которое будем рассматривать как одноточечное дифференциальное включение, гамильтониан которого $H=\psi(f(x, t)+g(x))$ имеет вид $(7.0)$.

Для $a>0$ положим $J_{a}=a x\left(t_{1}\right)-x\left(t_{0}\right)$ и рассмотрим задачу:

$$
J_{a} \rightarrow \max , \quad \dot{x}=f(x, t)+g(x)
$$

Поставим вопрос: для каких $а$ траектория $x^{0}(t), t \in\left[t_{0}, t_{1}\right]$, удовлетворяет необходимьм условиям $(6.12 \mathrm{c}),\left(6.12 \mathrm{~d}^{\prime}\right),\left(6.12 \mathrm{~d}^{\prime \prime}\right)$. Напомним, что условие $\left(6.12 \mathrm{~d}^{\prime \prime}\right)$ мы рассматриваем для двух последовательностей (пр) и (лев). Соответствующие им условия будем обозначать $\left(6.12 \mathrm{~d}^{\prime \prime}\right)-п р ~ и ~\left(6.12 \mathrm{~d}^{\prime \prime}\right)-$-л.

В теории оптимального управления для классических задач с управляемыми системами доказано [1], что условия принципа максимума и укороченного принципа максимума эквивалентны. Однако в задачах с дифференциальными включениями условия $\left(6.12 \mathrm{~d}^{\prime}\right)$ и $\left(6.12 \mathrm{~d}^{\prime \prime}\right)$ независимы друг от друга. Покажем это на рассматриваемой задаче для последовательности (лев).

Рассмотрим сначала случай $\gamma_{k}^{1}=1, \gamma_{k}^{2}=\gamma>0$. Заметим, что согласно условию трансверсальности $\psi^{0}\left(t_{0}\right)=1, \psi^{0}\left(t_{1}\right)=a$, и поэтому $\psi^{0}(t)>0 \forall t \in$ $\left[t_{0}, t_{1}\right]$.

В данном случае имеем

$$
\stackrel{\circ}{\mathscr{H}}_{\mathrm{Cl}}\left(\psi^{0}(t), x^{0}(t), t ; \bar{\psi}, \bar{x}, \bar{t}\right)=\bar{\psi} g\left(x^{0}(t)\right)+\psi^{0}(t)(2|\bar{x}|+\gamma|\bar{t}|) .
$$

Отсюда условие $\left(6.12 \mathrm{~d}^{\prime}\right)$ с учетом (6.12c) примет вид:

$$
\left|\frac{\dot{\psi}^{0}(t)}{\psi^{0}(t)}\right| \leqslant 2, \quad\left|\frac{\dot{\mathscr{H}}^{0}(t)}{\psi^{0}(t)}\right| \leqslant \gamma
$$


г де $\mathscr{H}^{0}(t)=\mathscr{H}\left(\psi^{0}(t), x^{0}(t), t\right)$. Подставляя сюда выражение $\dot{\mathscr{H}}{ }^{0}=\dot{\psi}^{0} g^{0}+\psi^{0} g_{x}^{0} g^{0}$, получаем

$$
\left|\frac{\dot{\psi}^{0}(t)}{\psi^{0}(t)}\right| \leqslant 2, \quad\left|\frac{\dot{\psi}^{0}}{\psi^{0}}+g_{x}\left(x^{0}(t)\right)\right| \leqslant \frac{\gamma}{g\left(x^{0}(t)\right)} .
$$

Обратимся к условию $\left(6.12 \mathrm{~d}^{\prime \prime}\right)$-л. По построению функции $f(x, t)$ для почти всех $t$ сушествует $\delta(t)>0$ такое, что

$$
f(x, t)=0 \text { для всех } x:\left|x-x^{0}(t)\right|<\delta(t) .
$$

Отсюда можно показать, что правая часть условия $\left(6.12 \mathrm{~d}^{\prime \prime}\right)-$ л равна $\psi^{0}(t)|\bar{x}|$. Следовательно, условие $\left(6.12 \mathrm{~d}^{\prime \prime}\right)$-л имеет вид:

$$
\left|\frac{\dot{\psi}^{0}(t)}{\psi^{0}(t)}\right| \leqslant 1
$$

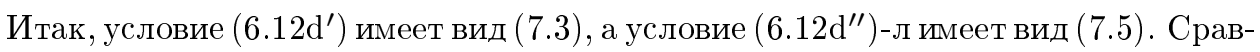
ним условия (7.3) и (7.5). Ясно, что при достаточно большом $\gamma$ условие (7.3) эквивалентно неравенству

$$
\left|\frac{\dot{\psi}^{0}(t)}{\psi^{0}(t)}\right| \leqslant 2 .
$$

Следовательно, при больших $\gamma$ условие (7.5) сильнее условия (7.3). Однако при малых $\gamma$ это уже не так. Покажем это.

Рассмотрим случай, когда $\gamma_{k}^{1} \rightarrow 0, \gamma_{k}^{2}=\gamma$. Тогда

$$
\stackrel{\circ}{\mathscr{H}}_{\mathrm{Cl}}\left(\psi^{0}(t), x^{0}(t), t ; \bar{\psi}, \bar{x}, \bar{t}\right)=\bar{\psi} g\left(x^{0}(t)\right)+\psi^{0}(t)(|\bar{x}|+\gamma|\bar{t}|)
$$

и условие $\left(6.12 \mathrm{~d}^{\prime}\right)$ с учетом (6.12c) примет вид:

$$
\left|\frac{\dot{\psi}^{0}}{\psi^{0}}\right| \leqslant 1, \quad\left|\frac{\dot{\psi}^{0}}{\psi^{0}}+g_{x}\left(x^{0}(t)\right)\right| \leqslant \frac{\gamma}{g\left(x^{0}(t)\right)} .
$$

Условие (6.12d")-л по-прежнему имеет вид (7.5). Снова сравним эти два условия теперь в виде (7.6) и (7.5). Ясно, что условие (7.5) в данном случае следует из условия (7.6), причем при малых $\gamma$ они не эквивалентны.

Итак, в системе условий (6.12a)-(6.12d") условия $\left(6.12 \mathrm{~d}^{\prime}\right)$ и $\left(6.12 \mathrm{~d}^{\prime \prime}\right)$-л действительно не следуют одно из другого. Наша же теорема 6.1 утверждает, что оба они должны иметь место, причем для одной и той же функции $\psi^{0}(t)$.

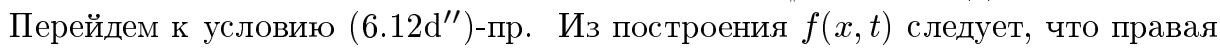
часть $\left(6.12 \mathrm{~d}^{\prime \prime}\right)$-пр равна нулю. Следовательно, $\dot{\mathscr{H}}^{0}(t)=0$, что эквивалентно условию:

$$
\frac{\dot{\psi}^{0}}{\psi^{0}}=-g_{x}\left(x^{0}(t)\right) .
$$

Интегрируя это равенство с учетом условия трансверсальности, получаем

$$
\psi^{0}(t)=\frac{g\left(x^{0}\left(t_{0}\right)\right)}{g\left(x^{0}(t)\right)} .
$$


Таким образом, $\psi^{0}(t)$ определяется по $\left(6.12 \mathrm{~d}^{\prime \prime}\right)$-пр однозначно и, следовательно, только при одном значении

$$
a=\frac{g\left(x^{0}\left(t_{0}\right)\right)}{g\left(x^{0}\left(t_{1}\right)\right)}
$$

можно ожидать, что траектория $x^{0}(t), t \in\left[t_{0}, t_{1}\right]$, будет решением задачи на $\max J_{a}$.

$\mathrm{K}$ тем же результатам мы легко придем, рассматривая гамильтониан $\mathscr{H}=$ $|\psi(f(x, t)+g(x))|$, что соответствует дифференциальному включению

$$
|\dot{x}| \leqslant|f(x, t)+g(x)| \text {. }
$$

\section{Часть II. Усиление условий Смирнова}

Мы по-прежнему рассматриваем задачу $Z$.

\section{§ 8. Формулировка результата}

ТЕОрема 8.1. Пусть $x^{0}(t)$ - сильный минимум задачи Z. Тогда имеет место следующая альтернатива: либо существуют $\alpha_{0}, \alpha \in \mathbb{R}^{d(h)}, \beta \in \mathbb{R}^{d(g)}$ такие, что

$$
\begin{gathered}
\alpha_{0} \geqslant 0, \quad \alpha \geqslant 0, \quad \alpha h\left(p^{0}\right)=0, \quad \alpha_{0}+|\alpha|+|\beta|>0 \\
l_{p}\left(p^{0}\right)=0, \quad \text { əде } \quad l(p)=\alpha_{0} J(p)+\alpha h(p)+\beta g(p)
\end{gathered}
$$

либо для любой последовательности $\nu^{m}(t)$ ядер осреднения по $t$ такой, что $\eta\left(\nu^{m}\right) \rightarrow 0$, существуют $\alpha_{0}, \alpha, \beta$ и абсолютно непрерывная функиия $\psi(t)$ такие, что:

a) $\alpha_{0} \geqslant 0, \alpha \geqslant 0, \alpha h\left(p^{0}\right)=0, \alpha_{0}+|\alpha|+|\beta|>0, \psi(t) \neq 0 \quad \forall t \in\left[t_{0}, t_{1}\right]$;

b) $\psi\left(t_{0}\right)=l_{x_{0}}\left(p^{0}\right), \psi\left(t_{1}\right)=-l_{x_{1}}\left(p^{0}\right), \psi(t) \dot{x}^{0}(t)=\mathscr{H}\left(\psi(t), x^{0}(t), t\right)$.

Кроме того, существует множсество полной меры, в каждой точке которого для любых $\bar{x}, \bar{t}$ выполнены неравенства:

c)

$$
\begin{aligned}
& \bar{x} \dot{\psi}(t)-\bar{t} \dot{\mathscr{H}}(t) \leqslant|\psi(t)| \varlimsup \overline{\lim }\left\{\rho_{x}\left(x^{\prime}, t^{\prime}, u^{\prime}\right) \bar{x}+\rho_{t}\left(x^{\prime}, t^{\prime}, u^{\prime}\right) \bar{t} \mid\right. \\
& \left.\quad\left(x^{\prime}, t^{\prime}, u^{\prime}\right) \in \Sigma, \quad\left(x^{\prime}, t^{\prime}, u^{\prime}, \rho_{u}\left(x^{\prime}, t^{\prime}, u^{\prime}\right)\right) \rightarrow\left(x^{0}(t), t, \dot{x}^{0}(t), \frac{\psi(t)}{|\psi(t)|}\right)\right\}
\end{aligned}
$$

d)

$$
\begin{aligned}
& \bar{x} \dot{\psi}(t)-\bar{t} \dot{\mathscr{H}}(t) \leqslant|\psi(t)| \overline{\lim }\left\{\rho_{x}\left(x^{\prime}, t^{\prime}, u^{\prime}\right) \bar{x}+\rho_{t}^{\nu^{m}}\left(x^{\prime}, t^{\prime}, u^{\prime}\right) \bar{t} \mid m \rightarrow \infty,\right. \\
& \left.\quad\left(x^{\prime}, u^{\prime}\right) \in \Sigma(t), \quad\left(x^{\prime}, u^{\prime}, \rho_{u}\left(x^{\prime}, t^{\prime}, u^{\prime}\right)\right) \rightarrow\left(x^{0}(t), \dot{x}^{0}(t), \frac{\psi(t)}{|\psi(t)|}\right), \frac{\eta\left(\nu^{m}\right)}{\rho\left(\sigma^{\prime}\right)} \rightarrow 0\right\} .
\end{aligned}
$$


Здесъ $\mathscr{H}(t)=\mathscr{H}\left(\psi(t), x^{0}(t), t\right)$,

$$
\begin{aligned}
& \Sigma=\{(x, t, u) \mid \rho(x, t, u)>0, \rho \text { обладает } \\
&\text { полньцм дифференциалом в точке }(x, t, u)\}, \\
& \Sigma(t)=\{(x, u) \mid \rho(x, t, u)>0, \rho \text { обладает } \\
&\text { дифференциалом по }(x, u) \text { в точке }(x, t, u)\} .
\end{aligned}
$$

Условия а), b), с) эквивалентны условиям Смирнова [3]. Условия а), b), с), d) сильнее условий Смирнова. Дальнейшее есть доказательство сформулированной теоремы.

\section{§9. Построение аппроксимирующей последовательности}

\section{1. Переход к автономной задаче.}

ЗАДАчА $\widehat{Z}$.

$$
\begin{gathered}
J(p) \rightarrow \min , \quad h(p) \leqslant 0, \quad g(p)=0, \\
\frac{d x}{d \tau}=u, \quad \frac{d t}{d \tau}=v, \quad \tau \in\left[\tau_{0}, \tau_{1}\right], \quad \text { где } \tau_{0}=t_{0}, \quad \tau_{1}=t_{1}, \\
P(v) \leqslant 0, \quad \text { где } P(v)=(v-1 / 2)(v-2), \\
\zeta \in F(x, t), \quad \text { где } \zeta=\frac{u}{v} .
\end{gathered}
$$

Положим $\widehat{t}(\tau)=\tau, \widehat{x}(\tau)=x^{0}(\widehat{t}(\tau))$. Нетрудно видеть, что $\widehat{x}(\tau), \widehat{t}(\tau)-$ сильньй минимум задачи $\widehat{Z}$. Дальнейшие рассмотрения мы проводим в предположении, что первая альтернатива теоремы 8.1 не имеет места.

Пусть задана последовательность $\nu^{m}(t), m=1,2, \ldots$, ядер осреднения по $t$ такая, что $\eta\left(\nu^{m}\right) \rightarrow 0$ при $m \rightarrow \infty$. Пусть $\mu^{m}(x)$ - последовательность ядер осреднения по $x$ такая, что $\eta\left(\mu^{m}\right) \rightarrow 0$ и $\eta\left(\nu^{m}\right) \int\left|\mu_{x}^{m}(x)\right| d x \rightarrow 0$ при $m \rightarrow \infty$. Очевидно, это требование корректно.

Положим $\gamma^{m}(x, t)=\mu^{m}(x) \cdot \nu^{m}(t)$ и

$$
\rho^{m}(x, t, \zeta)=\int d x^{\prime} d t^{\prime}\left[\rho\left(x^{\prime}, t^{\prime}, \zeta\right) \cdot \gamma^{m}\left(x^{\prime}-x, t^{\prime}-t\right)\right]=\rho^{\gamma^{m}}(x, t, \zeta) .
$$

9.2. Первая аппроксимирующая последовательность. Выберем последовательность $\eta^{m}$ из условий: $\eta^{m} \rightarrow 0, \eta\left(\gamma^{m}\right)=o\left(\eta^{m}\right)$ при $m \rightarrow \infty$. Тогда согласно предложению 3.3 последовательность

$$
U^{m}(x, t)=\left\{u \mid \rho^{\gamma^{m}}(x, t, u) \leqslant \eta^{m}\right\}
$$

приближается сверху к отображению $F(x, t)$.

Обозначим далее

$$
\widehat{p}=\left(\widehat{x}\left(\tau_{0}\right), \widehat{x}\left(\tau_{1}\right)\right), \quad \widehat{u}(\tau)=\frac{d}{d \tau} \widehat{x}(\tau), \quad \widehat{v}(\tau)=\frac{d}{d \tau} \widehat{t}(\tau) .
$$

Очевидно,

$$
\widehat{p}=p^{0}, \quad \widehat{v}(\tau)=1, \quad \tau \in\left[\tau_{0}, \tau_{1}\right]
$$

Определим задачу: 
ЗАДАчА $\widehat{Z}^{m}$.

$$
\begin{gathered}
J(p)+(p-\widehat{p})^{2}+\int_{\tau_{0}}^{\tau_{1}}\left((u-\widehat{u})^{2}+(v-\widehat{v})^{2}\right) d \tau \rightarrow \min , \\
h(p) \leqslant 0, \quad g(p)=0, \quad t\left(\tau_{0}\right)=t_{0}, \quad t\left(\tau_{1}\right)=t_{1}, \\
\frac{d x}{d \tau}=u, \quad \frac{d t}{d \tau}=v, \quad P(v) \leqslant 0, \\
\rho^{m}(x, t, \zeta) \leqslant \eta^{m}, \quad \zeta=\frac{u}{v} .
\end{gathered}
$$

По теореме 2.2 для каждого достаточно большого $m$ сушествует пара $\left(\widehat{x}^{m}(\tau), \widehat{t}^{m}(\tau)\right)$ - сильный минимум задачи $\widehat{Z}^{m}-$ такая, что $\left(\widehat{x}^{m}(\tau), \widehat{t}^{m}(\tau)\right) \rightarrow$ $(\widehat{x}(\tau), \widehat{t}(\tau))$ при $m \rightarrow \infty$ равномерно на $\left[\tau_{0}, \tau_{1}\right]$, а $\left(\widehat{u}^{m}(\tau), \widehat{v}^{m}(\tau)\right) \rightarrow(\widehat{u}(\tau), \widehat{v}(\tau))$ в смысле нормы $L_{2}$ на $\left[\tau_{0}, \tau_{1}\right]$.

9.3. Вторая аппроксимирующая последовательность. Пусть функция $s^{m}(\tau)$ определена равенством:

$$
\widehat{t}^{m}\left(s^{m}(\tau)\right)=\tau \text { на }\left[\tau_{0}, \tau_{1}\right]
$$

Обозначим $\frac{d s^{m}}{d \tau}$ через $\omega^{m}(\tau)$. Задача $\widetilde{Z}^{m}$ определяется для тех $m$, для которых определены $\widehat{x}^{m}(\tau), \widehat{t}^{m}(\tau)$, и имеет вид:

ЗАДАчА $\widetilde{Z}^{m}$.

$$
\begin{gathered}
J(p)+(p-\widehat{p})^{2}+\int_{\tau_{0}}^{\tau_{1}}\left[\left(\frac{u(\tau)}{\omega^{m}(\tau)}-\widehat{u}\left(s^{m}(\tau)\right)\right)^{2}+\left(\frac{v(\tau)}{\omega^{m}(\tau)}-1\right)^{2}\right] \omega^{m}(\tau) d \tau \rightarrow \min , \\
h(p) \leqslant 0, \quad g(p)=0, \quad t\left(\tau_{0}\right)=\tau_{0}, \quad t\left(\tau_{1}\right)=\tau_{1}, \\
\frac{d x}{d \tau}=u, \quad \frac{d t}{d \tau}=v, \\
P\left(\frac{v(\tau)}{\omega^{m}(\tau)}\right) \leqslant 0, \quad \rho(x, t, \zeta) \leqslant \eta^{m}, \quad \zeta=\frac{u}{v} .
\end{gathered}
$$

Положим $\widetilde{x}^{m}(\tau)=\widehat{x}^{m}\left(s^{m}(\tau)\right), \widetilde{t}^{m}(\tau)=\widehat{t}^{m}\left(s^{m}(\tau)\right)$. Тогда $\widetilde{x}^{m}(\tau), \widetilde{t}^{m}(\tau)-$ сильный минимум задачи $\widetilde{Z}^{m}$. Заметим, что $\widetilde{t}^{m}(\tau)=\tau$. Заметим также, что

$$
\omega^{m}(\tau) \stackrel{L_{2}}{\longrightarrow} 1 \text { при } m \rightarrow \infty,
$$

ибо

$$
\begin{gathered}
\int\left(\widehat{v}^{m}\left(s^{m}(\tau)\right)-1\right)^{2} d s^{m}(\tau) \rightarrow 0, \\
\widehat{v}^{m}\left(s^{m}(\tau)\right) \omega^{m}(\tau)=1, \quad \omega^{m}(\tau) \in[1 / 2,2] .
\end{gathered}
$$

Отсюда

$$
\widetilde{u}^{m}(\tau) \stackrel{L_{2}}{\longrightarrow} \widehat{u}(\tau), \quad \widetilde{x}^{m} \stackrel{C}{\longrightarrow} \widehat{x}(\tau) \text { при } m \rightarrow \infty .
$$

В силу предложения 3.2 задача $\widetilde{Z}^{m}$ является регулярной задачей оптимального управления. И таким образом, для всех $m$ траектория $\left(\widetilde{x}^{m}(\tau), \tau, \widetilde{t}^{m}(\tau), 1\right)$ удовлетворяет соответствуюшему ПМ. 


\section{§10. Принцип максимума для траектории $\widetilde{x}^{m}(\tau), \widetilde{t}^{m}(\tau)$}

Принцип максимума состоит в следуюшем: существуют постоянные $\alpha_{0}^{m}, \beta_{m}^{0}$, $\beta_{1}^{m}$, векторы $\alpha^{m} \in \mathbb{R}^{d(h)}, \beta^{m} \in \mathbb{R}^{d(y)}$, измеримые скалярные функции $\lambda_{1}^{m}(\tau)$, $\lambda_{2}^{m}(\tau)$, абсолютно непрерывная функция $\psi^{m}(\tau)$ со значениями в $\mathbb{R}^{d(x)}$, абсолютно непрерывная скалярная функция $\psi_{t}^{m}(\tau)$ такие, что выполнены следующие условия:

a) $\alpha_{0}^{m} \geqslant 0, \alpha^{m} \geqslant 0, \alpha^{m} h^{m}(\widetilde{p})=0, \alpha_{0}^{m}+\left|\alpha^{m}\right|+\left|\beta^{m}\right|+\left|\beta_{0}^{m}\right|+\left|\beta_{1}^{m}\right|=1$;

b) $\lambda_{1}^{m}(\tau)\left\{\begin{array}{ll}\geqslant 0, & \tau \in \mathscr{E}_{1}^{m}, \\ =0, & \tau \notin \mathscr{E}_{1}^{m},\end{array} \quad \lambda_{2}^{m}(\tau)\left\{\begin{array}{ll}\geqslant 0, & \tau \in \mathscr{E}_{2}^{m}, \\ =0, & \tau \notin \mathscr{E}_{2}^{m},\end{array} \quad\right.\right.$ где

$$
\begin{aligned}
& \mathscr{E}_{1}=\left\{\tau \mid \rho^{m}\left(\widetilde{x}^{m}(\tau), \widetilde{t}^{m}(\tau), \widetilde{\zeta}^{m}(\tau)\right)=\eta^{m}\right\}, \\
& \mathscr{E}_{2}=\left\{\tau \mid P\left(\frac{\widetilde{v}^{m}(\tau)}{\omega^{m}(\tau)}\right)=0\right\}
\end{aligned}
$$

(в дальнейшем для сокращения положим $\sigma=(x, t, \zeta))$;

c)

$$
\begin{aligned}
& -2 \alpha_{0}^{m}\left(\frac{\widetilde{u}^{m}(\tau)}{\omega^{m}(\tau)}-\widehat{u}\left(s^{m}(\tau)\right)\right)+\psi^{m}(\tau)= \begin{cases}\lambda_{1}^{m}(\tau) \rho_{\zeta}^{m}\left(\widetilde{\sigma}^{m}(\tau)\right) \cdot \frac{1}{\widetilde{v}^{m}(\tau)}, & \tau \in \mathscr{E}_{1}^{m}, \\
0, & \tau \notin \mathscr{E}_{1}^{m},\end{cases} \\
& -2 \alpha_{0}^{m}\left(\frac{\widetilde{v}^{m}(\tau)}{\omega^{m}(\tau)}-1\right)+\psi_{t}^{m}(\tau) \\
& = \begin{cases}-\lambda_{1}^{m}(\tau) \rho_{\zeta}^{m}\left(\widetilde{\sigma}^{m}(\tau)\right) \cdot \frac{\widetilde{\zeta}^{m}(\tau)}{\widetilde{v}^{m}(\tau)}+\lambda_{2}^{m}(\tau) P^{\prime}\left(\frac{\widetilde{v}^{m}(\tau)}{\omega^{m}(\tau)}\right) \frac{1}{\omega^{m}(\tau)}, & \tau \in \mathscr{E}_{1}^{m}, \\
\lambda_{2}^{m}(\tau) P^{\prime}\left(\frac{\widetilde{v}^{m}(\tau)}{\omega^{m}(\tau)}\right) \frac{1}{\omega^{m}(\tau)}, & \tau \notin \mathscr{E}_{1}^{m},\end{cases}
\end{aligned}
$$

где $P^{\prime}(z)=2 z-5 / 2$;

d)

$$
\begin{aligned}
& \frac{d \psi^{m}}{d \tau}=\lambda_{1}^{m}(\tau) \rho_{x}^{m}\left(\widetilde{\sigma}^{m}(\tau)\right), \\
& \frac{d \psi_{t}^{m}}{d \tau}=\lambda_{1}^{m}(\tau) \rho_{t}^{m}\left(\widetilde{\sigma}^{m}(\tau)\right) ;
\end{aligned}
$$

e) $\psi^{m}\left(\tau_{0}\right)=l_{x_{0}}^{m}\left(\widetilde{p}^{m}\right), \psi^{m}\left(\tau_{1}\right)=-l_{x_{1}}^{m}\left(\widetilde{p}^{m}\right), \psi_{t}^{m}\left(\tau_{0}\right)=\beta_{0}^{m}, \psi_{t}^{m}\left(\tau_{1}\right)=\beta_{1}^{m}$, где $l^{m}(p)=\alpha_{0}^{m} J(p)+\alpha^{m} h(p)+\beta^{m} g(p)+(p-\widehat{p})^{2} \alpha_{0}^{m}$.

\section{§11. Предварительный анализ принципа максимума}

Положим

$$
\varepsilon^{m}=\frac{c_{0} \eta\left(\sigma^{m}\right)}{\eta^{m}\left(1-c_{0} \eta\left(\sigma^{m}\right) / \eta^{m}\right)},
$$

тогда из предложения 3.2 и (3.17) имеем

$$
1 \geqslant\left|\rho_{\zeta}^{m}\left(\widetilde{\sigma}^{m}(\tau)\right)\right| \geqslant 1-\sqrt{2 \varepsilon^{m}}, \quad \tau \in \mathscr{E}_{1}^{m} .
$$


Положим

$$
\begin{aligned}
& \delta_{u}^{m}(\tau)=-2 \alpha_{0}^{m}\left(\frac{\widetilde{u}^{m}(\tau)}{\omega^{m}(\tau)}-\widehat{u}\left(s^{m}(\tau)\right)\right) \\
& \delta_{v}^{m}(\tau)=-2 \alpha_{0}^{m}\left(\frac{\widetilde{v}^{m}(\tau)}{\omega^{m}(\tau)}-1\right)
\end{aligned}
$$

тогда из условия е) $\S 10$, из (3.16) и (11.1) при $\tau \in \mathscr{E}_{1}^{m}$ следует:

$$
\lambda_{1}^{m}(\tau) \leqslant \frac{1}{1-\sqrt{2 \varepsilon^{m}}}\left(\left|\delta_{u}^{m}(\tau)\right|+\left|\psi^{m}(\tau)\right|\right) .
$$

Очевидно, что эта оценка верна всюду на $\left[\tau_{0}, \tau_{1}\right]$, ибо $\lambda_{1}^{m}(\tau)=0$ при $\tau \notin \mathscr{E}_{1}^{m}$.

Заметим, с другой стороны, что $\left|\rho_{x}^{m}(\sigma)\right| \leqslant c_{0} \forall \sigma$, где $c_{0}$ - постоянная Липшица отображения $F$. Тогда согласно условию d) $\S 10$ и (11.2)

$$
\left|\frac{d \psi^{m}}{d \tau}\right| \leqslant \frac{c_{0}}{1-\sqrt{2 \varepsilon^{m}}}\left(\left|\delta_{u}^{m}(\tau)\right|+\left|\psi^{m}(\tau)\right|\right)
$$

Из условий а), е) $\S 10$ следует, что последовательность $\psi^{m}\left(\tau_{0}\right)$ ограничена. Далее, по определению $\widetilde{u}^{m}(\tau)=\widehat{u}^{m}\left(s^{m}(\tau)\right) \omega^{m}(\tau)$. Отсюда следует, что последовательности $\delta_{u}^{m}(\tau), \delta_{v}^{m}(\tau)$ равномерно ограничены. Тогда из (11.2) и (11.3) следует равномерная ограниченность последовательностей $\lambda_{1}^{m}(\tau), \psi^{m}(\tau), \frac{d \psi^{m}}{d \tau}$. Отсюда согласно d) $\S 10$ и оценке $\left|\rho_{t}^{m}(\sigma)\right| \leqslant c_{0} \forall \sigma$ получим, что последовательности $\psi_{t}^{m}(\tau)$, $\frac{d \psi_{t}^{m}}{d \tau}$ также равномерно ограничены.

Из второго условия с) $\S 10$ получим теперь, что последовательность $\lambda_{2}^{m}(\tau)$ равномерно ограничена. Но

$$
\frac{\widetilde{v}^{m}(\tau)}{\omega^{m}(\tau)}=\frac{1}{\omega^{m}(\tau)} \stackrel{L_{2}}{\longrightarrow} 1 \text { при } m \rightarrow \infty
$$

Следовательно, mes $\mathscr{E}_{2}^{m} \rightarrow 0$ и тогда

$$
\int_{\tau_{0}}^{\tau_{1}} \lambda^{m}(\tau) d \tau \rightarrow 0, \quad m \rightarrow \infty
$$

Теперь мы докажем неравенство

$$
\underset{m \rightarrow \infty}{\lim _{m}}\left(\alpha_{0}^{m}+\left|\alpha^{m}\right|+\left|\beta^{m}\right|\right)>0 .
$$

Допустим, что существует подпоследовательность $m_{k}$ такая, что $\alpha_{0}^{m_{k}}+\left|\alpha^{m_{k}}\right|+$ $\left|\beta^{m_{k}}\right| \rightarrow 0$ при $k \rightarrow \infty$. Тогда согласно е) $\S 10 \psi^{m_{k}}\left(\tau_{0}\right) \rightarrow 0$. Далее, $\delta_{u}^{m_{k}} \stackrel{L_{2}}{\longrightarrow} 0$ при $k \rightarrow \infty$. Тогда из (11.3) следует: $\psi^{m_{k}}(\tau) \stackrel{C}{\longrightarrow} 0$ при $k \rightarrow \infty$. Получим $\lambda_{1}^{m_{k}}(\tau) \stackrel{L_{2}}{\longrightarrow} 0$ при $k \rightarrow \infty$.

Далее, из неравенства $1 / 2 \leqslant \omega^{m}(\tau) \leqslant 2,(9.2)$ и $(9.3)$ следует: $\delta_{u}^{m}(\tau) \stackrel{L_{2}}{\longrightarrow} 0$ при $m \rightarrow \infty$. Тогда из с) $\S 10,(11.2)$ и (11.4) будем иметь: $\psi_{t}^{m_{k}}(\tau) \stackrel{L_{2}}{\longrightarrow} 0$ при $k \rightarrow \infty$. 
Но последовательность $\frac{d \psi_{t}^{m}}{d \tau}$ равномерно ограничена. Поэтому $\psi_{t}^{m_{k}}(\tau) \stackrel{C}{\longrightarrow} 0$ при $k \rightarrow \infty$. Отсюда получаем $\beta_{0}^{m_{k}}, \beta_{1}^{m_{k}} \rightarrow 0$ при $k \rightarrow \infty$, что противоречит условию a) $\S 10$. Тем самым (11.5) доказано.

\section{§ 12. Необходимые условия}

12.1. Переход к пределу в принципе максимума. Выберем подпоследовательность $\pi^{0} \in\{m\}$ из условий: существуют $\widehat{\psi}(\tau), \widehat{\psi}_{t}(\tau)$ такие, что почти всюду на $\left[\tau_{0}, \tau_{1}\right]$

$$
\begin{gathered}
\widehat{\psi}(\tau)=\lim _{\pi^{0}} \psi^{m}(\tau), \quad \widehat{p}_{t}(\tau)=\lim _{\pi^{0}} \psi_{t}^{m}(\tau), \\
\widetilde{u}(\tau) \rightarrow \widehat{u}(\tau), \quad \omega^{m}(\tau) \rightarrow 1, \quad m \in \pi^{0},
\end{gathered}
$$

и при этом

$$
\alpha_{0}^{m}, \alpha^{m}, \beta^{m} \rightarrow \alpha_{0}, \alpha, \beta, \quad m \in \pi^{0}
$$

соответственно. Эти условия осушествимы: условие (12.1) в силу предкомпактности в $C$ последовательностей $\psi^{m}(\tau), \psi_{t}^{m}(\tau)$; условие $(12.2)$ в силу $(9.2)$ и $(9.3)$; условие (12.3) в силу условия а) $\S 10$.

Итак, подпоследовательность $\pi^{0}$ выбрана. Согласно выбору $\delta_{u}^{m}(\tau), \delta_{v}^{m}(\tau) \rightarrow 0$, $m \in \pi^{0}$, почти всюду. Тогда из (11.3) имеем:

$$
\left|\frac{d \widehat{\psi}}{d \tau}\right| \leqslant c_{0}|\widehat{\psi}(\tau)|
$$

Отсюда следует, что либо $\widehat{\psi}(\tau)=0$ для любого $\tau$, либо $\widehat{\psi}(\tau) \neq 0$ для любого $\tau$. Первая возможность не реализуется в силу предположения о том, что первая альтернатива теоремы 8.1 не имеет места. Действительно, согласно условию е) $§ 10$ имеем $\widehat{\psi}\left(\tau_{0}\right)=l_{x_{0}}(\widehat{p}), \widehat{\psi}\left(\tau_{1}\right)=-l_{x_{1}}(\widehat{p})$, где $l(p)=\alpha_{0} J(p)+\alpha h(p)+\beta g(p)$ и, кроме того, $\alpha h(\widehat{p})=0$. Согласно (11.5) имеем $\alpha_{0}+|\alpha|+|\beta|>0$, а поскольку $\widehat{p}=p^{0}$, то если мы допустим, что $\widehat{\psi}(\tau)=0$ для любого $\tau$, тем самым мы допустим, что первая альтернатива теоремы 8.1 реализуется. Итак, $\widehat{\psi}(\tau) \neq 0$ для любого $\tau \in\left[\tau_{0}, \tau_{1}\right]$.

Имеем из первого условия с) $\S 10$ и (12.2):

$$
\widehat{\psi}(\tau)=\lim _{\pi^{0}} \lambda_{1}^{m}(\tau) \rho_{\zeta}^{m}\left(\widetilde{\sigma}^{m}(\tau)\right) \text { почти всюду на }\left[\tau_{0}, \tau_{1}\right]
$$

Следовательно,

$$
\lim _{\pi^{0}} \lambda_{1}^{m}(\tau)=|\widehat{\psi}(\tau)|, \quad \lim _{\pi^{0}} \rho_{\zeta}^{m}\left(\widetilde{\sigma}^{m}(\tau)\right)=\frac{\widehat{\psi}(\tau)}{|\widehat{\psi}(\tau)|}
$$

почти всюду на $\left[\tau_{0}, \tau_{1}\right]$.

Отсюда и из (12.5) получим

$$
\begin{gathered}
\widehat{\psi}(\tau) \widehat{u}(\tau)=|\widehat{\psi}(\tau)| \lim _{\pi^{0}} \rho_{\zeta}^{m}\left(\widetilde{\sigma}^{m}(\tau)\right) \widetilde{\zeta}^{m}(\tau), \\
\widehat{\psi}_{t}(\tau)=-\lim _{\pi^{0}}|\widehat{\psi}(\tau)| \rho_{\zeta}^{m}\left(\widetilde{\sigma}^{m}(\tau)\right) \widetilde{\zeta}^{m}(\tau) .
\end{gathered}
$$


Отсюда видно, что

$$
\widehat{\psi}(\tau) \widehat{u}(\tau)+\widehat{\psi}_{t}(\tau)=0
$$

Но согласно (3.17)

$$
\rho_{\zeta}^{m}\left(\widetilde{\sigma}^{m}(\tau)\right)-\rho_{\zeta}\left(\widetilde{\sigma}^{m}(\tau)\right) \rightarrow 0
$$

Тогда из (12.7) получим, опираясь на формулу двойственности $\left(3.2^{\prime}\right)$,

$$
-\widehat{\psi}_{t}(\tau)=|\widehat{\psi}(\tau)| \lim _{\pi^{0}} \rho\left(\widetilde{\sigma}^{m}(\tau)\right)+\mathscr{H}\left(\rho_{\zeta}\left(\widetilde{\sigma}^{m}(\tau)\right), \widetilde{x}^{m}(\tau), \widehat{t}(\tau)\right),
$$

откуда согласно (12.6), (12.7) и (12.9)

$$
\begin{gathered}
\widehat{\psi}_{t}(\tau)=-\mathscr{H}(\widehat{\psi}(\tau), \widehat{x}(\tau), \widehat{t}(\tau)), \\
\widehat{\psi}(\tau) \widehat{u}(\tau)=\mathscr{H}(\widehat{\psi}(\tau), \widehat{x}(\tau), \widehat{t}(\tau)), \\
\lim _{\pi^{0}} \rho_{\zeta}\left(\widetilde{\sigma}^{m}(\tau)\right)=\frac{\widehat{\psi}(\tau)}{|\widehat{\psi}(\tau)|}
\end{gathered}
$$

Перейдем к сопряженному уравнению, т.е. к условиям d) $§ 10$. Имеем

$$
\frac{d \psi^{m}}{d \tau} \stackrel{* \text {-сл }}{\longrightarrow} \frac{d \widehat{p}}{d \tau}, \quad \frac{d \psi_{t}^{m}}{d \tau} \stackrel{\text { *-сл }}{\longrightarrow} \frac{d \widehat{\psi}_{t}}{d \tau} \quad \text { при } \quad m \rightarrow \infty, \quad m \in \pi^{0} .
$$

По лемме 1.1 имеем

$$
\bar{x} \frac{d \widehat{\psi}}{d \tau}-\bar{t} \frac{d \widehat{\mathscr{H}}}{d \tau} \leqslant|\widehat{\psi}(\tau)| \overline{\lim }\left\{\rho_{x}^{m}\left(\widetilde{\sigma}^{m}(\tau)\right) \bar{x}+\rho_{t}^{m}\left(\widetilde{\sigma}^{m}(\tau)\right) \bar{t}\right\}, \quad m \rightarrow \infty, \quad m \in \pi^{0} .
$$

Это неравенство выполнено при любых $\bar{x}, \bar{t}$ для почти всех $\tau \in\left[\tau_{0}, \tau_{1}\right]$.

Заметим, что для почти всех $\tau$ начиная с некоторого $m(\tau)$ выполнено равенство $\rho^{m}\left(\widetilde{\sigma}^{m}(\tau)\right)=\eta^{m}$. Это следует из условия с) $\S 10,(12.6)$ и из условия: $\widehat{\psi}^{m} \neq 0$ для любого $\tau$.

Мы ограничимся в дальнейшем рассмотрением именно таких $\tau$. Обозначим это множество через $\mathscr{E}$. Пусть $\tau \in \mathscr{E}$. Тогда

$$
\begin{gathered}
\rho_{x}^{m}\left(\widetilde{\sigma}^{m}(\tau)\right) \bar{x}+\rho_{t}^{m}\left(\widetilde{\sigma}^{m}(\tau)\right) \bar{t} \\
=\int d x^{\prime} d t^{\prime}\left[\left(\rho_{x}^{m}\left(x^{\prime}, t^{\prime} ; \widetilde{\xi}^{m}(\tau)\right) \bar{x}+\rho_{t}^{m}\left(x^{\prime}, t^{\prime} ; \widetilde{\xi}^{m}(\tau)\right) \bar{t}\right) \gamma^{m}\left(x^{\prime}-\widetilde{x}^{m}(\tau), t^{\prime}-\widehat{t}(\tau)\right)\right]
\end{gathered}
$$

Пусть $x^{\prime}, t^{\prime}$ таковы, что

$$
\left|x^{\prime}-\widetilde{x}^{m}(\tau)\right| \leqslant \eta\left(\mu^{m}\right), \quad\left|t^{\prime}-\widehat{t}(\tau)\right| \leqslant \eta\left(\nu^{m}\right) .
$$

Так как $\tau \in \mathscr{E}$, то для достаточно больших $m \in \pi^{0}$ будет выполнено неравенство

$$
\rho\left(x^{\prime}, t^{\prime} ; \widetilde{\xi}^{m}(\tau)\right) \geqslant \eta^{m}-c_{0} \eta\left(\gamma^{m}\right)>0 \text {. }
$$


Отсюда следует, что правая часть (12.12) оценивается сверху для больших $m$ через

$$
\begin{gathered}
\sup \left\{\rho_{x}^{m}\left(\widetilde{\sigma}^{m}(\tau)\right) \bar{x}+\rho_{t}^{m}\left(\widetilde{\sigma}^{m}(\tau)\right) \bar{t} \mid\right. \\
\left.\left(x^{\prime}, t^{\prime} ; \widetilde{\xi}^{m}(\tau)\right) \in \Sigma,\left|x^{\prime}-\widetilde{x}^{m}(\tau)\right| \leqslant \eta\left(\mu^{m}\right),\left|t^{\prime}-\widehat{t}(\tau)\right| \leqslant \eta\left(\nu^{m}\right)\right\} .
\end{gathered}
$$

Заметим, что согласно (12.13) для всех достаточно больших $m$ имеет место неравенство (см. 3 ):

$$
\left|\rho_{u}\left(x^{\prime}, t^{\prime} ; \widetilde{\xi}^{m}(\tau)\right)-\rho_{u}^{m}\left(\widetilde{x}^{m}(\tau), \widehat{t}(\tau) ; \widetilde{\xi}^{m}(\tau)\right)\right| \leqslant \sqrt{2 \frac{c_{0} \eta\left(\gamma^{m}\right)}{\eta^{m}-c_{0} \eta\left(\gamma^{m}\right)}}
$$

Отсюда, из (12.2) и (12.14) следует, что правая часть (12.11) оценивается сверху через

$$
|\widehat{\psi}(\tau)| \varlimsup\left\{\rho_{x}\left(\sigma^{\prime}\right) \bar{x}+\rho_{t}\left(\sigma^{\prime}\right) \bar{t} \mid \sigma^{\prime} \rightarrow \widehat{\sigma}(\tau), \sigma^{\prime} \in \Sigma, \rho_{u}\left(\sigma^{\prime}\right) \rightarrow \frac{\widehat{\psi}(\tau)}{|\widehat{\psi}(\tau)|}\right\}
$$

Следовательно, из (12.11) получим: для всех $\bar{x}, \bar{t}$ на множестве полной меры выполнено неравенство

$\bar{x} \frac{d \widehat{\psi}}{d \tau}-\bar{t} \frac{d \widehat{\mathscr{H}}}{d \tau} \leqslant|\widehat{\psi}(\tau)| \overline{\lim }\left\{\rho_{x}\left(\sigma^{\prime}\right) \bar{x}+\rho_{t}\left(\sigma^{\prime}\right) \bar{t} \mid \sigma^{\prime} \rightarrow \widehat{\sigma}(\tau), \sigma^{\prime} \in \Sigma, \rho_{u}\left(\sigma^{\prime}\right) \rightarrow \frac{\widehat{\psi}(\tau)}{|\widehat{\psi}(\tau)|}\right\}$.

Теперь подойдем к неравенству $(12.11)$ с другой стороны. Для любого $\sigma=(x, t, \zeta)$ оценим $\left|\delta^{m}\right|$, где $\delta^{m}=\rho_{x}^{m}(\sigma)-\rho_{x}^{\mu^{m}}(\sigma)$. Имеем:

$$
\delta^{m}=\left(\rho^{\nu^{m}}\right)_{x}^{\mu^{m}}(\sigma)-\rho_{x}^{\mu^{m}}(\sigma)=\int d x^{\prime}\left[\mu_{x}^{m}\left(x^{\prime}-x\right)\left(\rho^{\nu^{m}}\left(x^{\prime}, t, \zeta\right)-\rho\left(x^{\prime}, t, \zeta\right)\right)\right] .
$$

Следовательно,

$$
\left|\delta^{m}\right| \leqslant c_{0} \eta\left(\nu^{m}\right) \int\left|\mu_{x}^{m}\left(x^{\prime}\right)\right| d x^{\prime} .
$$

Отсюда вытекает согласно выбору последовательности $\mu^{m}$, что $\delta^{m} \rightarrow 0$, причем равномерно по $\tau$. Тогда правую часть (12.11) можно, не нарушая неравенства (12.11), заменить на

$$
|\widehat{\psi}(\tau)| \varlimsup \overline{\lim }\left\{\rho_{x}^{\mu^{m}}\left(\widetilde{\sigma}^{m}(\tau)\right) \bar{x}+\rho_{t}^{m}\left(\widetilde{\sigma}^{m}(\tau)\right) \bar{t} \mid m \rightarrow \infty, m \in \pi^{0}\right\} .
$$

Имеем:

$$
\begin{gathered}
\rho_{x}^{\mu^{m}}\left(\widetilde{\sigma}^{m}(\tau)\right) \bar{x}+\rho_{t}^{m}\left(\widetilde{\sigma}^{m}(\tau)\right) \bar{t} \\
=\int d x^{\prime}\left[\mu^{m}\left(x^{\prime}-\widetilde{\sigma}^{m}(\tau)\right)\left(\rho_{x}\left(x^{\prime}, \widehat{t}(\tau), \widetilde{\xi}^{m}(\tau)\right) \bar{x}+\rho_{t}^{\nu^{m}}\left(x^{\prime}, \widehat{t}(\tau), \widetilde{\xi}^{m}(\tau)\right) \bar{t}\right)\right] \\
\leqslant \sup \left\{\rho_{x}\left(x^{\prime}, \widehat{t}(\tau), \widetilde{\xi}^{m}(\tau)\right) \bar{x}+\rho_{t}^{\nu^{m}}\left(x^{\prime}, \widehat{t}(\tau), \widetilde{\xi}^{m}(\tau)\right) \bar{t} \mid\right. \\
\left.\quad\left(x^{\prime}, \widehat{t}(\tau), \widetilde{\xi}^{m}(\tau)\right) \in \Sigma(\widehat{t}(\tau)),\left|x^{\prime}-\widetilde{x}^{m}(\tau)\right| \leqslant \eta\left(\mu^{m}\right)\right\} .
\end{gathered}
$$


Если $\tau \in \mathscr{E}$, а $m \in \pi^{0}$ и достаточно велико, то из неравенства $\left|x^{\prime}-\widetilde{x}^{m}(\tau)\right| \leqslant \eta\left(\mu^{m}\right)$ следует:

$$
\begin{gathered}
\rho\left(x^{\prime}, \widehat{t}(\tau), \widetilde{\xi}^{m}(\tau)\right) \geqslant \eta^{m}-c_{0} \eta\left(\mu^{m}\right), \\
\left|\rho_{u}\left(x^{\prime}, \widehat{t}(\tau) ; \widetilde{\xi}^{m}(\tau)\right)-\rho_{u}^{m}\left(\widetilde{\sigma}^{m}(\tau)\right)\right| \leqslant \sqrt{2 \frac{c_{0} \eta\left(\mu^{m}\right)}{\eta^{m}-c_{0} \eta\left(\mu^{m}\right)}} .
\end{gathered}
$$

Следовательно, при $m \rightarrow \infty, m \in \pi_{0}$ имеем для $\tau \in \mathscr{E}:$

$$
\begin{aligned}
& \eta\left(\mu^{m}\right)=o\left(\rho\left(x^{\prime}, \widehat{t}(\tau), \widetilde{\xi}^{m}(\tau)\right)\right), \\
& \rho_{u}\left(x^{\prime}, \widehat{t}(\tau) ; \widetilde{\xi}^{m}(\tau)\right) \rightarrow \frac{\widehat{\psi}(\tau)}{|\widehat{\psi}(\tau)|} .
\end{aligned}
$$

Тогда из (12.17) следует оценка сверху правой части (12.11):

$$
\begin{gathered}
|\widehat{\psi}(\tau)| \varlimsup\left\{\rho_{x}\left(\sigma^{\prime}\right) \bar{x}+\rho_{t}^{\nu^{m}}\left(\sigma^{\prime}\right) \bar{t} \mid \sigma^{\prime} \rightarrow \sigma^{0}(\tau), m \rightarrow \infty, \sigma^{\prime} \in \Sigma(\widehat{t}(\tau)),\right. \\
\left.\rho_{u}\left(\sigma^{\prime}\right) \rightarrow \frac{\widehat{\psi}(\tau)}{|\widehat{\psi}(\tau)|}, \eta\left(\nu^{m}\right)=o\left(\rho\left(\sigma^{\prime}\right)\right)\right\} .
\end{gathered}
$$

Таким образом, из (12.11) следует неравенство, справедливое для всех $\bar{x}, \bar{t}$ на множестве полной меры на $\left[\tau_{0}, \tau_{1}\right]$ :

$$
\begin{aligned}
\bar{x} \frac{d \widehat{\psi}}{d \tau}-\bar{t} \frac{d \widehat{\mathscr{H}}}{d \tau} \leqslant|\widehat{\psi}(\tau)| \varlimsup & \left\{\rho_{x}\left(\sigma^{\prime}\right) \bar{x}+\rho_{t}^{\nu^{m}}\left(\sigma^{\prime}\right) \bar{t} \mid \sigma^{\prime} \rightarrow \sigma^{0}(\tau)\right. \\
& \left.m \rightarrow \infty, \sigma^{\prime} \in \Sigma(\widehat{t}(\tau)), \rho_{u}\left(\sigma^{\prime}\right) \rightarrow \frac{\widehat{\psi}(\tau)}{|\widehat{\psi}(\tau)|}, \eta\left(\nu^{m}\right)=o\left(\rho\left(\sigma^{\prime}\right)\right)\right\} .
\end{aligned}
$$

Нам осталось перейти к переменной $t$ в условиях (12.15) и (12.18). Обращая функцию $\widehat{t}(\tau)$, получаем функцию $\widehat{\tau}(t)=t$ на $\left[t_{0}, t_{1}\right]$. Подставляя в $(12.15),(12.18)$ $\tau=\widehat{\tau}(t)$ и полагая $\psi^{0}(t)=\widehat{\psi}(\widehat{\tau}(t))$, получаем окончательно два неравенства, справедливые для любых $\bar{x}, \bar{t}$ при почти всех $t \in\left[t_{0}, t_{1}\right]$ :

$$
\begin{gathered}
\bar{x} \dot{\psi}^{0}(t)-\bar{t} \dot{\mathscr{H}}^{0}(t) \leqslant\left|\psi^{0}(t)\right| \varlimsup \lim \left\{\rho_{x}\left(\sigma^{\prime}\right) \bar{x}+\rho_{t}\left(\sigma^{\prime}\right) \bar{t} \mid \sigma^{\prime} \rightarrow \sigma^{0}(t),\right. \\
\left.\sigma^{\prime} \in \Sigma(t), \rho_{u}\left(\sigma^{\prime}\right) \rightarrow \frac{\psi^{0}(t)}{\left|\psi^{0}(t)\right|}\right\} \\
\bar{x} \dot{\psi}^{0}(t)-\bar{t} \dot{\mathscr{H}}^{0}(t) \leqslant\left|\psi^{0}(t)\right| \overline{\lim }\left\{\rho_{x}\left(\sigma^{\prime}\right) \bar{x}+\rho_{t}^{\nu^{m}}\left(\sigma^{\prime}\right) \bar{t} \mid \sigma^{\prime} \rightarrow \sigma^{0}(t), m \rightarrow \infty\right. \\
\left.\sigma^{\prime} \in \Sigma(t), \rho_{u}\left(\sigma^{\prime}\right) \rightarrow \frac{\psi^{0}(t)}{\left|\psi^{0}(t)\right|}, \eta\left(\nu^{m}\right)=o\left(\rho\left(\sigma^{\prime}\right)\right)\right\} .
\end{gathered}
$$

Здесь $\sigma^{0}(t)=\left(\lambda^{0}(t), t, x^{0}(t)\right)$.

Тем самьм теорема 8.1 полностью доказана. 


\section{Зак лючение}

Задачи негладкого оптимального управления не допускают вариационного исследования. Его заменяет выбор приближающих задач и необходимых условий для них с последующим рассмотрением предельного перехода.

Однако разные реализации этого плана приводят к разным условиям, причем ни одно из них не эквивалентно потере управляемости. Их содержание полностью исчерпывается формулировкой.

С другой стороны, в оптимальном управлении в продолжение линии Понтрягина любое необходимое условие (принцип максимума) эквивалентно потере управляемости в том или ином смысле и, таким образом, не допускает усиления. Для сравнения заметим, что условие Кларка слабее условия Смирнова, а оно, в свою очередь, слабее одного из наших условий.

Таким образом, аналога принципа максимума в негладком оптимальном управлении пока нет. И последнее. В настоящей работе мы опирались на принцип максимума для регулярных смешанных ограничений. Это позволило нам не только получить с единых позиций условия Кларка и Смирнова, но и усилить их. Однако в негладком оптимальном управлении не принято пользоваться достижениями понтрягинского оптимального управления, исключая классический принцип максимума Понтрягина и принцип максимума с фазовьми ограничениями Дубовицкого-Милютина - весьма давние результаты. Такое положение может лишш затормозить прогресс. Думается, что следует исходить из обратного: чем сильнее опираться на достижения понтрягинского оптимального управления, тем успешнее можно развивать негладкое оптимальное управление.

\section{Список литературы}

1. Афанасьев А.П., Дикусар В. В., Милютин А.А., Чуканов С. В. Необходимоеусловие в оптимальном управлении. Гл. I-V. М.: Наука, 1990.

2. Кларк Ф. Оптимизация и негладкий анализ. М.: Мир, 1988.

3. Смирнов Г. В. Дискретные аптроксимации и оптимальные решения диффференциальных включений // Кибернетика. 1991. № 1. С. 76-79.

Институт химической физики РАН, г. Москва
Поступила в редакцию 12.02.2001 и 09.08.2002 